\title{
Genome-wide comprehensive analysis of transcriptomes and small RNAs offers insights into the molecular mechanism of alkaline stress tolerance in a citrus rootstock
}

\author{
Juxun $\mathrm{Wu}^{1}$, Junying $\mathrm{CaO}^{1}$, Mei Su${ }^{1}$, Guizhi Feng ${ }^{1}$, Yanhui $\mathrm{Xu}^{1}$ and Hualin $\mathrm{Yi}^{1}$
}

\begin{abstract}
Alkaline stress has serious-negative effects on citrus production. Ziyang xiangcheng (Citrus junos Sieb. ex Tanaka) (Cj) is a rootstock that is tolerant to alkaline stress and iron deficiency. Trifoliate orange (Poncirus trifoliata (L.) Raf.) (Pt), the most widely used rootstock in China, is sensitive to alkaline stress. To investigate the molecular mechanism underlying the tolerance of $\mathrm{Cj}$ to alkaline stress, next-generation sequencing was employed to profile the root transcriptomes and small RNAs of $\mathrm{Cj}$ and Pt seedlings that were cultured in nutrient solutions along a three $\mathrm{pH}$ gradient. This two-level regulation data set provides a system-level view of molecular events with a precise resolution. The data suggest that the auxin pathway may play a central role in the inhibitory effect of alkaline stress on root growth and that the regulation of auxin homeostasis under alkaline stress is important for the adaptation of citrus to alkaline stress. Moreover, the jasmonate (JA) pathway exhibits the opposite response to alkaline stress in $\mathrm{Cj}$ and Pt and may contribute to the differences in the alkaline stress tolerance and iron acquisition between $\mathrm{Cj}$ and Pt. The dataset provides a wealth of genomic resources and new clues to further study the mechanisms underlying alkaline stress resistance in $\mathrm{Cj}$.
\end{abstract}

\section{Introduction}

Saline-alkaline soils are widespread throughout the world, limiting agricultural productivity globally ${ }^{1-3}$. Of the $\sim 831$ million ha of saline-alkaline soils, more than half are alkalinized $^{4,5}$. Under alkaline stress, most plants cannot survive because of the high $\mathrm{pH}$. Alkaline stress has a much stronger inhibitory effect on plant growth than salt stress $^{2,3,6}$. However, research examining the effect of alkaline stress on plants and the adaptation mechanism of plants to alkaline stress are much scarcer than research on salt stress ${ }^{2,7}$. Thus, elucidation of the molecular mechanisms of plant responses to alkaline stress is urgently needed.

\footnotetext{
Correspondence: Hualin Yi (yihualin@mail.hzau.edu.cn)

${ }^{1}$ Key Laboratory of Horticultural Plant Biology, Ministry of Education, Huazhong Agricultural University, Wuhan 430070, PR China
}

The root is an organ for the uptake and transport of water and nutrients, and it is also the first organ that perceives soil stresses. Root branching through lateral root (LR) formation is important for the root system to cope with various abiotic stresses ${ }^{8,9}$. Many studies have revealed that auxin acts as an integrator of many endogenous and exogenous signals for lateral root development regulation ${ }^{9}$. For instance, cytokinin (CK) inhibits LR formation by reducing auxin transport mediated by $P I N 1^{10}$ and by upregulating $M I Z 1^{11}$, a gene involved in the hydrotropism that reduces auxin accumulation in the roots. ABA can inhibit LR formation by upregulating miR393, which can suppress the expression of the TIR1/ AFB auxin receptor ${ }^{12}$. Shkolnik-Inbar and Bar-Zvi ${ }^{13}$ have shown that $\mathrm{ABA}$ and $\mathrm{CK}$ inhibit LR formation by

\section{(c) The Author(s) 2019}

(c) (i) Open Access This article is licensed under a Creative Commons Attribution 4.0 International License, which permits use, sharing, adaptation, distribution and reproduction c. in any medium or format, as long as you give appropriate credit to the original author(s) and the source, provide a link to the Creative Commons license, and indicate if changes were made. The images or other third party material in this article are included in the article's Creative Commons license, unless indicated otherwise in a credit line to the material. If material is not included in the article's Creative Commons license and your intended use is not permitted by statutory regulation or exceeds the permitted use, you will need to obtain permission directly from the copyright holder. To view a copy of this license, visit http://creativecommons.org/licenses/by/4.0/. 
upregulating the $A B I 4$ transcription factor via reducing polar auxin transport, leading to a reduction in LR development. In addition, many nutrients also influence LR development by interfering with the auxin pathway, such as nitrates ${ }^{14}$, potassium ${ }^{15}$, and phosphate ${ }^{16}$.

Jasmonate (JA) is a significant signaling substance that is involved in plant responses to several abiotic and biotic stresses, such as drought stress, salt stress, wounding (mechanical stress), and pathogen infection ${ }^{17}$. JA is also involved in the regulation of developmental processes, including root growth, pollen production and senescence $^{18}$. Many studies have shown that JA plays a positive role in salt stress responses in plants ${ }^{19-25}$. However, few studies have examined the role of JAs in response to alkaline stress compared with those on salt stress. Recently, Zhu et al. $^{26}$ reported that TIFY10 proteins, which are JAZ proteins, positively regulate plant alkaline stress responses. A similar result was obtained by Zhu et $\mathrm{al}^{27}{ }^{27}$, who reported that the overexpression of GsJAZ2 in Arabidopsis results in enhanced plant tolerance to salt and alkaline stress. In addition, JAs play important roles in root growth regulation. In Arabidopsis, treatment with JA can inhibit primary root growth ${ }^{28}$, whereas JA promotes lateral root formation at submicromolar concentrations by directly inducing the auxin biosynthesis gene $A S A 1$ and/or by modulating the PIN2 protein ${ }^{29,30}$. Raya-Gonzalez et al. $^{31}$ also showed that low concentrations of JA inhibit primary root growth and promote lateral root formation in Arabidopsis seedlings. In rice, RSS3 interacts with the JA pathway to play a significant role in the regulation of root cell elongation under stressful conditions ${ }^{32}$.

Generally, alkaline stress accompanies iron deficiency. Iron is required for essential everyday processes in plants and is abundant in most soils. However, most iron exists as $\mathrm{Fe}^{3+}$ hydroxides, which are only somewhat soluble at neutral $\mathrm{pH}$. The release of protons from roots increases the solubility of the $\mathrm{Fe}^{3+}$ hydroxides around the rhizosphere. The $\mathrm{Fe}^{3+}$ is reduced to $\mathrm{Fe}^{2+}$ by $\mathrm{FRO}^{33}$, which was the rate-limiting step for iron acquisition from the soil ${ }^{34}$. Iron is then transported into the root epidermal cells by IRT $1^{35,36}$. The FER-LIKE IRON DEFICIENCY-INDUCED TRANSCRIPTION FACTOR (FIT) plays a central role in upregulating the root-expressed genes involved in iron acquisition, which regulates $F R O 2$ at the transcription level and IRT1 at the protein level ${ }^{37}$. Indeed, a fit Arabidopsis mutant fails to take up iron and develops a lethal Fe deficiency, leading to leaf chlorosis ${ }^{38}$.

Citrus is one of the most important economic fruit crops worldwide. Grafting is the most important reproduction mode for citrus in the production industry. Moreover, rootstocks are significant for scions in citrus and can influence the fruit quality, canopy size, and resistance, among other aspects ${ }^{39}$. Ziyang xiangcheng (Citrus junos Sieb. ex Tanaka) (abbreviated Cj) is a special citrus germplasm native to China that is widely used as an iron-deficiency tolerant and alkaline-tolerant citrus rootstock in areas of China with calcareous soil. Trifoliate orange (Poncirus trifoliata (L.) Raf.) (abbreviated Pt) is the most widely used rootstock in China. However, Pt is sensitive to alkaline stress. Scions grafted on Pt show nutritional deficiency and growth retardation phenotypes in calcareous soil. However, little is known about the molecular mechanism underlying the differences in phenotypes between $\mathrm{Cj}$ and $\mathrm{Pt}$ regarding alkaline stress. Thus, in this study, the seedlings of $\mathrm{Cj}$ and Pt cultured in different $\mathrm{pH}$ nutrient solutions were used to perform comparative analyses, including assessments of the transcriptomes and small RNAs.

\section{Results}

Difference between $\mathrm{Cj}$ and Pt grown hydroponically with different $\mathrm{pH}$ gradients

After 8 weeks of culture in nutrient solutions of different $\mathrm{pH}$ (6.5, 8.0, and 9.5), $\mathrm{Cj}$ and Pt showed different performances. As shown in Fig. 1a, b, the lengths of the stems and roots were markedly decreased in both $\mathrm{Cj}$ and $\mathrm{Pt}$ under extremely alkaline conditions ( $\mathrm{pH}$ 9.5). At $\mathrm{pH}$ 6.5 , the lengths of the total roots and stems did not differ between the $\mathrm{Cj}$ and $\mathrm{Pt}$, but the number of lateral roots and total root surface area of the $\mathrm{Cj}$ were significantly increased compared with those of the Pt (Fig. 1b). As the $\mathrm{pH}$ value increased, the root architecture was altered in both $\mathrm{Cj}$ and $\mathrm{Pt}$, and the differences gradually increased between $\mathrm{Cj}$ and $\mathrm{Pt}$, including the total root and stem lengths, total root surface area and number of lateral roots (Fig. 1b). In particular, the number of lateral roots of $\mathrm{Cj}$ was much higher than that of Pt (approximately fourfold higher at $\mathrm{pH} 8.0$ and $\mathrm{pH}$ 9.5) (Fig. 1a, b).

The mineral elements in $\mathrm{Cj}$ and $\mathrm{Pt}$ were also measured. As shown in Fig. 1c, the contents of all of the mineral elements exhibited significant differences between $\mathrm{Cj}$ and Pt in the root tissue and mixed tissue (stem and leaf) under all three conditions. In the root tissue, the levels of most mineral elements exhibited a decreasing trend as the $\mathrm{pH}$ increased, with the exception of $\mathrm{Cu}, \mathrm{Na}$, and $\mathrm{Mg}$, and the trend curves of the mineral elements were similar for the $\mathrm{Cj}$ and $\mathrm{Pt}$. The $\mathrm{Na}$ and $\mathrm{Mg}$ levels increased as the $\mathrm{pH}$ value increased. The $\mathrm{Cu}$ levels increased with increasing $\mathrm{pH}$ in $\mathrm{Cj}$, whereas they decreased in Pt (Fig. 1c). Notably, the Fe levels in both tissues of the $\mathrm{Cj}$ were much higher than those in the Pt under all three conditions, and the $\mathrm{Fe}$ levels in both the $\mathrm{Cj}$ and Pt markedly decreased under alkaline stress (Fig. 1c).

\section{Global analysis of root transcriptomes, small RNAs, and degradomes of $\mathrm{Cj}$ and $\mathrm{Pt}$}

To investigate the underlying molecular changes that accompanied the morphological and physiological 

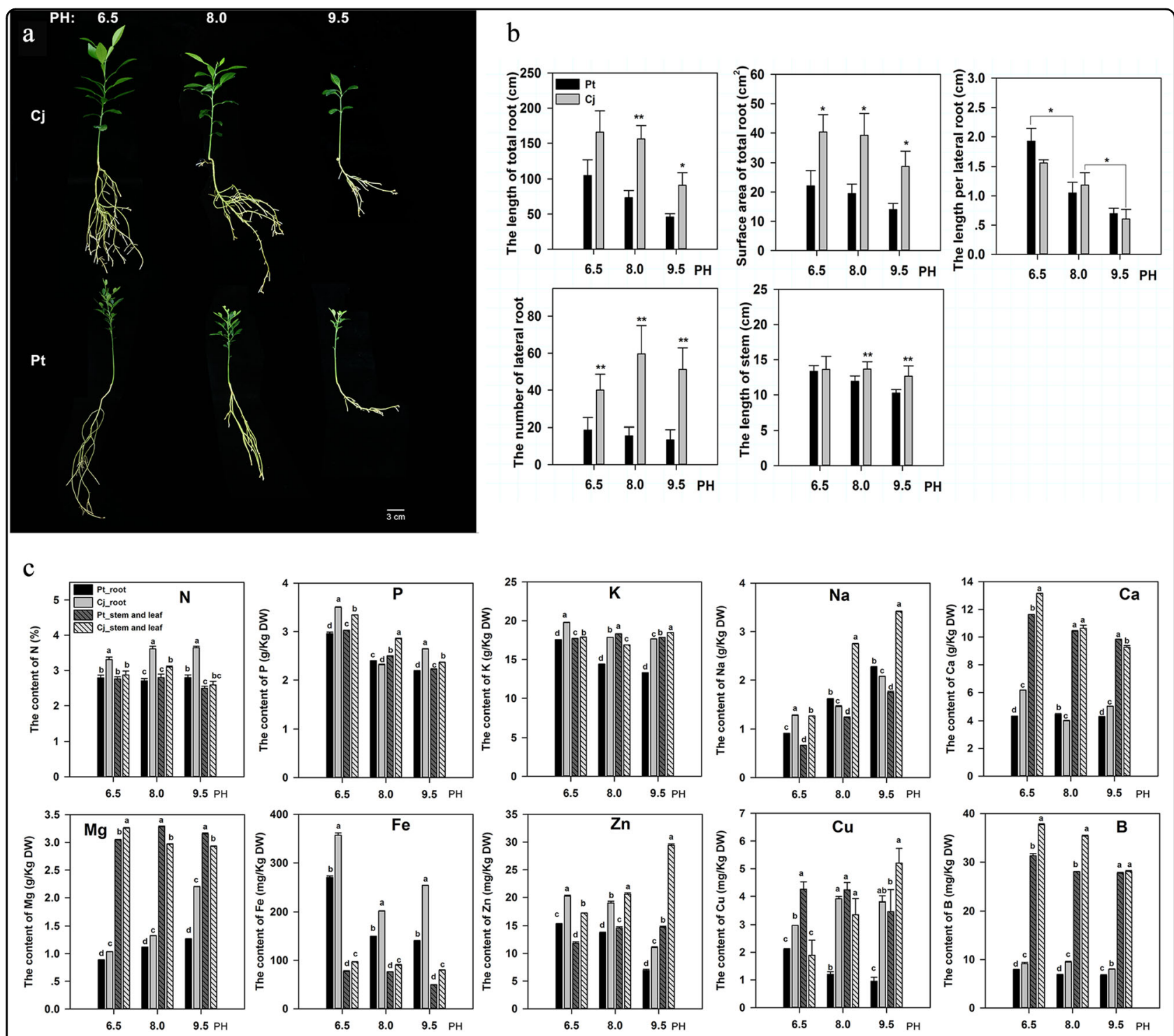

Fig. 1 Phenotypic characterization of seedlings of $\mathbf{C j}$ and $\mathrm{Pt}$ cultured in solutions along a $\mathbf{p H}$ gradient. a The morphological differences between $\mathrm{Cj}$ and Pt along a pH gradient $\mathbf{b}$ and the trends of several mineral element levels in the root, stem and leaf of $\mathrm{Cj}$ and $\mathrm{Pt}$ along a pH gradient c. In this figure, the samples are named "Sample_tissue.". Pt_root indicates the root tissue of Pt. Bars represent the standard error ( $n=3$ ). A

single asterisk $\left(^{*}\right)$ represents statistically significant differences $(P<0.05)$, and double asterisks $\left(^{* *}\right)$ represent highly statistically significant differences $(P<0.01)$, analyzed using Student's $t$-test. Lowercase represents statistically significant differences $(P<0.05)$, analyzed using one-way ANOVA

changes described above, we used RNA-seq to generate transcriptome, small RNA and degradome profiles of the root tissues of $\mathrm{Cj}$ and Pt. All sequencing data are summarized in Supplementary Table S1. Regarding the transcriptomes, after the low-quality reads had been removed, 40-55 million clean reads per sample were mapped against the Citrus sinensis reference genome ${ }^{40}$ (Supplementary Table S1). The correlation dendrogram illustrates the global relative relationships among the 18 transcriptomes (Fig. 2a). All three biological replicates clustered together, indicating good repeatability of the RNA-seq data. The $\mathrm{Cj}$ and $\mathrm{Pt}$ samples were clustered together, respectively (Fig. 2a). A total of 28,470 genes were identified in these six samples (Supplementary Table S2). Between 19,348 (Pt3) and 20,674 (Cj1) genes were detected in each sample (Fig. 2b). In total, 18,019 genes were common among all six samples (Fig. 2b, Supplementary Table S2).

For small RNAs, a total of 18 small RNA libraries were constructed for six samples. Each sample was assessed in the three biological replicates. In total, 11-26 million clean reads per replicate were obtained by RNA-seq (Supplementary Table S1). A search of the Rfam, Silva, GtRNAdb, and Repbase databases was used to remove 




structural RNAs (rRNAs, tRNAs, scRNAs, snRNAs, and snoRNAs). Unannotated sRNAs (4.3-16.4 million reads) were mapped to the Citrus sinensis genome and were used to identify known miRNAs and predict novel miRNAs by miRDEEP $2^{41}$ based on the structure and expression criteria $^{42}$ (Supplementary Table S1). In total, 49 known miRNAs and 52 novel miRNAs were identified in the $\mathrm{Cj}$ and Pt samples (Supplementary Table S8 and Figure S5).

To identify the target genes of the miRNAs, degradome sequencing ${ }^{43,44}$ was performed to experimentally validate the target genes of the miRNAs by capturing mRNA cleavage segments. To maximize the identification of the miRNA targets, we pooled RNA from $\mathrm{Cj} 1, \mathrm{Cj} 2$, and $\mathrm{Cj} 3$ into one $\mathrm{Cj}$ library and pooled RNA from Pt1, Pt2, and Pt3 into one Pt library. In total, 27.04 and 26.15 million clean reads were obtained from the $\mathrm{Cj}$ and $\mathrm{Pt}$ libraries, respectively (Supplementary Table S1). After removing miRNAs, structural RNAs and others, 16.06 and 15.88 million reads of the $\mathrm{Cj}$ and $\mathrm{Pt}$, respectively, were mapped to the Citrus sinensis genome (Supplementary Table S1). The reads that mapped to the cDNA (the reads in the cDNA_sense category in Supplementary Table S1) were analyzed to detect candidate targets of miRNAs.

\section{Identification of temporal expression trends accompanying gradient alkaline stress}

STEM $^{45}$ was used to perform clustering to identify the different gene expression profiles across all three $\mathrm{pH}$ conditions in the gradient. After the STEM clustering algorithm was applied, 16 model profiles with 5 statistically significant model profiles (colored profiles) were identified in $\mathrm{Cj}$, and 16 model profiles with 7 statistically significant model profiles were identified in Pt (Fig. 2c, d, Supplementary Table S3). Model profiles with the same color belonged to the same cluster of profiles. Therefore, two cluster profiles (an upregulated profile and downregulated profile) were identified in $\mathrm{Cj}$ and $\mathrm{Pt}$, respectively (Fig. 2c, d). These four cluster profiles were named $\mathrm{Cj}$ up, 
Cj down, Pt up, and Pt down. A total of 506, 859, 950, and 1417 genes were clustered into the $\mathrm{Cj}$ up, $\mathrm{Cj}$ down, Pt up, and $\mathrm{Pt}$ down profiles, respectively (Fig. 2c, d). A Venn diagram revealed that only 157 genes exhibited common expression trends in $\mathrm{Cj}$ and $\mathrm{Pt}$ (Supplementary Figure S1a). This result revealed that large differences existed between $\mathrm{Cj}$ and $\mathrm{Pt}$ at the transcription level in response to alkaline stress.

These four gene sets ( $\mathrm{Cj}$ up, $\mathrm{Cj}$ down, Pt up, and $\mathrm{Pt}$ down) were subject to KEGG pathway enrichment analysis (Supplementary Table S4). As shown in Fig. 2c, d, the top 10 over-represented KEGG pathways are listed. Notably, the "Phenylpropanoid biosynthesis" and "Plant hormone signal transduction" pathways were enriched in all four gene sets. Then, we used a heatmap to display the gene expression trends of the genes that were enriched in these two pathways (Supplementary Figure S2). As shown in Figure S2a, b, in the phenylpropanoid biosynthesis pathway, 22 peroxidase genes $(22 / 50)$ were downregulated in $\mathrm{Pt}$ with increasing $\mathrm{pH}$ values, whereas only 4 peroxidase genes $(4 / 18)$ were downregulated in $\mathrm{Cj}$. In the plant hormone signal transduction pathway, many auxin signal transduction genes (14 genes in $\mathrm{Pt}$ and 10 genes in $\mathrm{Cj}$ ) were enriched (Supplementary Figure S2c, d). In Pt, most auxin signal transduction genes (12/14) were downregulated. In contrast, one-half of the auxin signal transduction genes $(5 / 10)$ were downregulated in $\mathrm{Cj}$. These results indicate that peroxidase and auxin signal transduction genes may play significant roles in response to alkaline stress.

In addition, we identified transcription factors (TFs) in these four profiles. In total, 66 and 97 TFs were identified in $\mathrm{Cj}$ and Pt, respectively (Supplementary Figure S1b, c, Table S3). Among these TFs, the number of ERF family TFs was considerably greater compared with the number of TFs in the other families (Supplementary Figure S1b, c). Moreover, 24 TFs were identified both in $\mathrm{Cj}$ and $\mathrm{Pt}$ (Supplementary Table S3). As shown in Supplementary Figure S1d, the expression trends of most of these 24 TFs differed between $\mathrm{Cj}$ and Pt. Dynamic expression changes associated with these TFs may reveal their key functions. For example, $M Y C 2$ (orange1.1t01021), GBF4 (orange1.1t01148), WRKY46 (orange1.1t00472), and ZAT12 (Cs8g17960) were specifically downregulated in $\mathrm{Cj}$ and upregulated in $\mathrm{Pt}$ as the $\mathrm{pH}$ value increased (Supplementary Figure S1d, Table S3). Notably, MYC2 is a key gene in JA signal transduction. This finding indicates that JA signaling may also be involved in response to alkaline stress.

\section{Analysis of transcriptomic changes in response to alkaline stress}

As alkaline stress increased, the phenotypes were largely altered in both $\mathrm{Cj}$ and Pt. Simultaneously, the transcriptome profiles of $\mathrm{Cj}$ and $\mathrm{Pt}$ were also significantly altered. In $\mathrm{Cj}, 503,681$, and 106 DEGs (differentially expressed genes) were identified in the $\mathrm{Cj} 1 \mathrm{vs}$. $\mathrm{Cj} 2, \mathrm{Cj} 1 \mathrm{vs}$. $\mathrm{Cj} 3$, and $\mathrm{Cj} 2$ vs. Cj3 comparison groups, respectively (Fig. 3a). In Pt, 98, 996, and 496 DEGs were identified in the Pt1 vs. Pt2, Pt1 vs. Pt3, and Pt2 vs. Pt3 comparison groups, respectively (Fig. 3c). The smallest difference was noted between the $\mathrm{Cj} 2$ and $\mathrm{Cj} 3$ transcriptomes as well as between the P1 and Pt2 transcriptomes. This result suggests that $\mathrm{Cj}$ responds to alkaline stress more quickly than Pt.

To further characterize the significant roles of $\mathrm{Cj}$ and $\mathrm{Pt}$ in response to alkaline stress, the DEGs of these six comparison groups of $\mathrm{Cj}$ and $\mathrm{Pt}$ were used to perform GO-based term and KEGG-based pathway enrichment analyses (Supplementary Tables S6 and S7). Because the number of enriched pathways in $\mathrm{Cj} 2$ vs. $\mathrm{Cj} 3$ and $\mathrm{P} 1$ vs. Pt2 was minimal, the pathways enriched in the other four comparison groups were used to identify important pathways. The top 10 most enriched pathways and the DEGs distributed in these pathways were visualized using the GOplot package ${ }^{46}$ (Fig. 3b, d). The number of upregulated DEGs was more than that of downregulated DEGs in the top 10 pathways in the $\mathrm{Cj} 1 \mathrm{vs}$. $\mathrm{Cj} 3$ group, whereas the opposite pattern was observed in the Pt1 vs. Pt3 group (Fig. 3b, d). Several key pathways were screened from these four comparison groups, including "Glucosinolate biosynthesis", "Phenylpropanoid biosynthesis", "Carotenoid biosynthesis", "alpha-Linolenic acid metabolism", and "Plant hormone signal transduction" (Fig. 3b, d). In the glucosinolate biosynthesis pathway, three genes (Cs7g29760, Cs7g29770, and orange1.1t01456) encoding phenylalanine $\mathrm{N}$-monooxygenase (CYP79A2) were enriched. These genes were all upregulated in $\mathrm{Cj}$ and downregulated in $\mathrm{Pt}$ as the $\mathrm{pH}$ increased. CYP79A2 is a key gene in the biosynthesis of glucotropaeolin ${ }^{47}$. The expression patterns of the DEGs enriched in "Phenylpropanoid biosynthesis", "Carotenoid biosynthesis", "alpha-Linolenic acid metabolism", and "Plant hormone signal transduction" were visualized by heatmaps (Fig. 4). As shown in Fig. 4, in the phenylpropanoid biosynthesis pathway, many peroxidase genes were enriched, displaying V-shaped expression patterns in $\mathrm{Cj}$. However, these genes displayed downregulation trends in Pt. Moreover, several key genes involved in JA, ABA, and strigolactone (SL) biosynthesis were significantly altered by alkaline stress, such as LOX3 (Cs1g17380) and JMT (Cs7g31430, Cs1g24440) for JA biosynthesis, NCED1 (Cs5g14370, Cs2g03270) and ABA 8'-hydroxylase 3 (Cs3g23530) for ABA biosynthesis, and D27 (Cs5g30540) for SL biosynthesis (Fig. 4, Table 1). According to the expression trends of these key genes, the levels of ABA, JA, and SL may be altered by alkaline stress. This result indicates that ABA, JA, and SL may play important roles in tolerance to alkaline stress. In addition, many DEGs were 


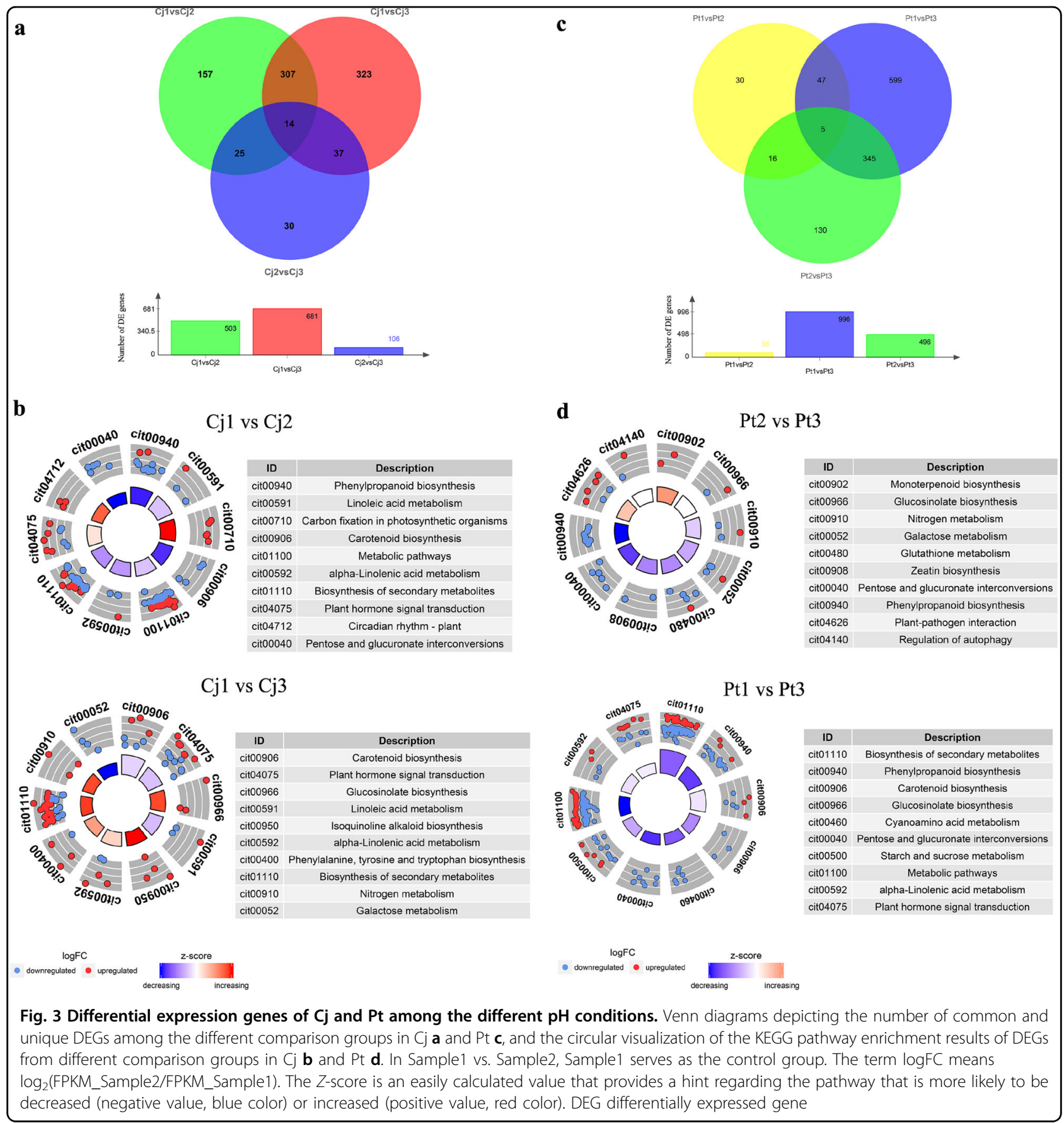

enriched in plant hormone signal transduction, including auxin, CK, ABA, JA, ethylene (ETH) and salicylic acid signals (Fig. 4). More DEGs were enriched in auxin signal transduction in both $\mathrm{Cj}$ and $\mathrm{Pt}$, and these DEGs displayed mixed expression patterns (some were upregulated, and some were downregulated). In $\mathrm{Pt}$, the number of downregulated auxin signal genes was greater than that of the upregulated genes, and the opposite finding was observed for $\mathrm{Cj}$. This result indicates that the auxin signal in $\mathrm{Pt}$ is more downregulated by alkaline stress compared with that in $\mathrm{Cj}$. Moreover, the JA, ABA, CK, and ETH signals were also altered by alkaline stress in both $\mathrm{Cj}$ and Pt (Fig. 4).

As shown in Fig. 1c, the uptake of mineral elements, especially metal elements such as $\mathrm{Fe}, \mathrm{Zn}$, and $\mathrm{Cu}$, is largely influenced by alkaline stress. The GO enrichment analysis results indicated that 49 and 68 DEGs were significantly enriched in the "ion transport" term in $\mathrm{Cj}$ and $\mathrm{Pt}$, respectively (Supplementary Figure S3, Table S7). As shown in Supplementary Figure S4a, iron-transport-related genes 


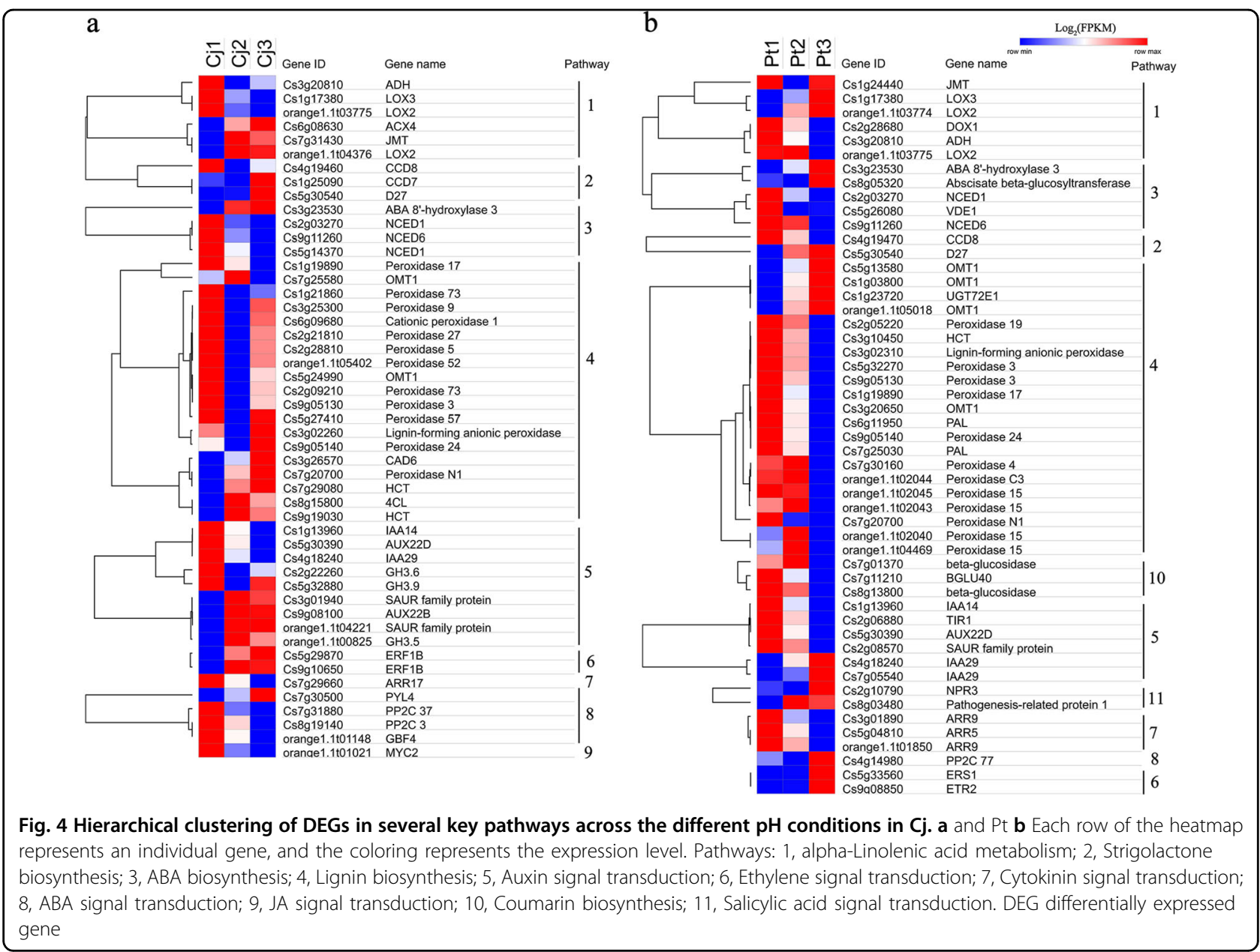

(FIT1, FRO2/4/6, Ferritin-1), zinc-transport-related genes (ZIP1/5/10), and copper-transport-related genes (COPT1) were upregulated in $\mathrm{Cj}$ under alkaline stress (Table 1). However, iron-transport-related genes (FIT1, FRO2/4, Ferritin1, and vacuolar iron transport 1) were largely downregulated at pH 9.5 in Pt (Supplementary Figure S4b, Table 1). Zinc-transport-related genes (ZIP5/10) and copper-transport-related genes (COPT1/6) were also upregulated in Pt under alkaline stress. In addition, six aquaporin genes (two TIP1-3s, two PIP2-2s, TIP2-2, and NIP2-1) were largely downregulated in Pt at pH 9.5 (Supplementary Figure S4b, Table 1). These results suggest that Pt may be more susceptible to iron and water deficiencies under alkaline stress.

\section{Comparing $\mathrm{Cj}$ and $\mathrm{Pt}$ transcriptomes}

$\mathrm{Cj}$ is more tolerant to alkaline stress compared with $\mathrm{Pt}$ in citrus production. To compare the differences of $\mathrm{Cj}$ and $\mathrm{Pt}$ at the transcriptome level, we identified some key pathways and genes involved in the response to alkaline stress. As shown in Fig. 5a, 4831, 4294 and 4742 DEGs were identified in the Pt1 vs. Cj1, Pt2 vs. Cj2 and Pt3 vs.
Cj3 comparison groups, respectively. The DEGs of these three groups were subject to GO-based term and KEGG-based pathway enrichment analyses (Supplementary Tables S6 and S7). The top 12 most enriched pathways and DEGs enriched in these pathways were visualized using the GOplot package ${ }^{46}$ (Fig. 5b). The top 4 most enriched pathways were the same in the three comparison groups. As the $\mathrm{pH}$ increased, the number of upregulated DEGs in the top 4 pathways increased (Fig. 5b). This result indicates that there are intrinsic differences in these pathways between $\mathrm{Cj}$ and $\mathrm{Pt}$, which are magnified under alkaline stress. In particular, the "Biosynthesis of secondary metabolites" pathway was largely altered by alkaline stress in both $\mathrm{Cj}$ and $\mathrm{Pt}$ (Figs. 3b, d and 5b). In addition, the "Phenylpropanoid biosynthesis", "alpha-Linolenic acid metabolism", "Carotenoid biosynthesis", and "Plant hormone signal transduction" remained enriched in these three or two comparison groups, which was consistent with the above result (Figs. 3b, d and 5b). In addition, the auxin biosynthesis pathway ("Tryptophan metabolism" pathway) was enriched in the Pt2 vs. Cj2 and Pt3 vs. Cj3 
Table 1 A list of important differentially expressed genes in $\mathrm{Cj}$ or $\mathrm{Pt}$ under different pH conditions

\begin{tabular}{|c|c|c|c|c|c|c|c|}
\hline \multirow[t]{2}{*}{ Gene ID } & \multirow[t]{2}{*}{ Gene name } & \multicolumn{6}{|l|}{ FPKM } \\
\hline & & $C_{j 1}$ & $\mathrm{Cj} 2$ & $\mathrm{Cj3}$ & Pt1 & Pt2 & $\mathrm{Pt} 3$ \\
\hline \multicolumn{8}{|c|}{ Plant hormone biosynthesis } \\
\hline Cs1g17380 & LOX3 & 126.20 & 60.53 & 43.40 & 51.93 & 76.67 & 177.22 \\
\hline orange1.1t03774 & LOX2 & 231.01 & 289.28 & 324.75 & 234.67 & 380.47 & 483.81 \\
\hline Cs1g24440 & $J M T$ & 4.87 & 8.52 & 5.89 & 79.69 & 37.49 & 77.35 \\
\hline Cs2g03270 & NCED1 & 1.42 & 0.55 & 0.45 & 3.31 & 1.45 & 0.87 \\
\hline Cs5g14370 & NCED1 & 59.44 & 19.47 & 7.08 & 19.29 & 12.78 & 22.50 \\
\hline Cs3g23530 & ABA 8'-hydroxylase 3 & 0.29 & 0.87 & 0.96 & 1.75 & 3.48 & 8.42 \\
\hline \multicolumn{8}{|c|}{ Auxin signal transduction } \\
\hline Cs2g06880 & TIR1 & 14.56 & 11.52 & 12.04 & 12.48 & 9.11 & 5.72 \\
\hline Cs1g13960 & IAA14 & 83.72 & 57.11 & 38.22 & 88.59 & 54.95 & 37.91 \\
\hline Cs3g01940 & SAUR family protein & 192.92 & 331.85 & 309.91 & 75.05 & 54.95 & 49.84 \\
\hline Cs4g18240 & IAA29 & 35.45 & 11.17 & 4.40 & 0.45 & 1.04 & 2.04 \\
\hline Cs5g30390 & $A \cup \times 22 D$ & 33.49 & 13.10 & 4.49 & 5.12 & 3.04 & 1.70 \\
\hline \multicolumn{8}{|c|}{ JA signal transduction } \\
\hline Cs1g17220 & TIFY $10 \mathrm{~A}$ & 72.55 & 78.69 & 62.94 & 187.85 & 179.28 & 190.65 \\
\hline Cs7g02820 & TIFY 9 & 104.68 & 109.34 & 71.07 & 267.47 & 279.69 & 337.96 \\
\hline orange1.1t01021 & MYC2 & 159.59 & 83.55 & 67.92 & 123.03 & 170.53 & 227.57 \\
\hline \multicolumn{8}{|c|}{ Cytokinin signal transduction } \\
\hline Cs5g04810 & ARR5 & 68.03 & 114.63 & 111.99 & 107.62 & 57.38 & 26.45 \\
\hline Cs3g01890 & ARR9 & 12.95 & 20.49 & 19.15 & 86.02 & 38.91 & 25.45 \\
\hline orange1.1t01850 & ARR9 & 24.43 & 46.81 & 44.90 & 131.95 & 84.08 & 35.45 \\
\hline \multicolumn{8}{|c|}{ ABA signal transduction } \\
\hline Cs7g30500 & PYL4 & 10.59 & 15.90 & 31.14 & 15.66 & 15.16 & 10.68 \\
\hline Cs7g31880 & PP2C 37 & 137.34 & 79.47 & 67.85 & 70.22 & 62.68 & 65.05 \\
\hline Cs4g14980 & PP2C 77 & 17.38 & 13.60 & 9.11 & 2.93 & 2.20 & 6.36 \\
\hline orange1.1t01148 & GBF4 & 25.27 & 17.10 & 11.24 & 7.30 & 11.06 & 14.95 \\
\hline \multicolumn{8}{|c|}{ ETH signal transduction } \\
\hline Cs5g33560 & ERS1 & 53.88 & 53.74 & 62.50 & 41.84 & 42.04 & 84.30 \\
\hline Cs9g08850 & ETR2 & 25.49 & 22.29 & 25.90 & 13.98 & 14.13 & 28.92 \\
\hline Cs9g10650 & ERF1B & 0.59 & 2.40 & 2.28 & 11.72 & 6.31 & 35.82 \\
\hline Cs5g29870 & ERF1B & 3.91 & 8.79 & 11.45 & 17.88 & 19.25 & 28.21 \\
\hline \multicolumn{8}{|l|}{ Ion transport } \\
\hline Cs6g09150 & Ferritin-1 & 308.96 & 263.21 & 132.31 & 69.33 & 74.37 & 8.59 \\
\hline Cs8g15600 & FIT1 & 10.02 & 14.90 & 25.94 & 5.30 & 5.71 & 1.88 \\
\hline orange1.1t00399 & FRO2 & 1.32 & 1.44 & 14.88 & 2.38 & 5.40 & 1.61 \\
\hline Cs9g19350 & FRO4 & 12.08 & 32.08 & 20.97 & 4.75 & 12.10 & 3.35 \\
\hline Cs3g01120 & FRO6 & 45.44 & 64.64 & 77.65 & 54.26 & 36.29 & 35.95 \\
\hline Cs2g17670 & Vacuolar iron transporter 1 & 7.96 & 8.24 & 6.91 & 3.24 & 6.01 & 1.08 \\
\hline
\end{tabular}


Table 1 continued

\begin{tabular}{|c|c|c|c|c|c|c|c|}
\hline \multirow[t]{2}{*}{ Gene ID } & \multirow[t]{2}{*}{ Gene name } & \multicolumn{6}{|l|}{ FPKM } \\
\hline & & Cj1 & $\mathrm{Cj} 2$ & Cj3 & Pt1 & Pt2 & Pt3 \\
\hline Cs2g17680 & Vacuolar iron transporter 1 & 1.03 & 0.87 & 0.88 & 2.59 & 4.52 & 0.39 \\
\hline Cs4g04460 & HKT1 & 8.73 & 4.83 & 3.48 & 13.89 & 12.01 & 6.33 \\
\hline Cs1g17440 & Potassium transporter & 1.71 & 3.46 & 6.56 & 5.03 & 5.39 & 1.97 \\
\hline Cs8g19470 & COPT1 & 105.38 & 280.36 & 146.19 & 239.85 & 307.49 & 89.99 \\
\hline Cs6g11670 & PIP2-2 & 1.68 & 1.79 & 2.14 & 247.81 & 211.03 & 61.79 \\
\hline Cs6g11700 & PIP2-2 & 742.85 & 515.42 & 512.74 & 327.20 & 237.80 & 98.21 \\
\hline Cs7g28650 & TIP1-3 & 318.73 & 206.19 & 209.54 & 865.85 & 888.13 & 374.02 \\
\hline Cs8g17900 & TIP1-3 & 525.44 & 309.47 & 308.31 & 445.38 & 478.62 & 193.00 \\
\hline Cs5g08710 & TIP2-2 & 249.87 & 159.79 & 208.14 & 290.55 & 318.31 & 129.69 \\
\hline Cs6g20570 & PMA1 & 93.09 & 84.97 & 103.70 & 71.72 & 80.69 & 156.91 \\
\hline Cs2g10720 & ZIP10 & 1.29 & 9.05 & 68.08 & 3.88 & 23.69 & 8.59 \\
\hline Cs2g11620 & ZIP5 & 17.59 & 34.08 & 62.11 & 58.95 & 59.75 & 92.42 \\
\hline Cs4g08930 & ZIP5 & 52.70 & 110.94 & 76.30 & 77.90 & 83.16 & 66.52 \\
\hline Cs6g11470 & ZIP5 & 71.45 & 130.31 & 184.01 & 77.77 & 123.44 & 232.80 \\
\hline \multicolumn{8}{|c|}{ Response to stimulus } \\
\hline orange1.1t03944 & ORG2 & 1.63 & 1.93 & 12.84 & 7.15 & 8.57 & 35.16 \\
\hline Cs1g11190 & ZFP1 & 4.63 & 9.85 & 12.34 & 16.25 & 15.37 & 5.34 \\
\hline Cs6g05660 & ZAT11 & 22.12 & 5.86 & 10.95 & 66.02 & 17.73 & 9.48 \\
\hline Cs1g18580 & DOF1.7 & 44.31 & 20.81 & 11.51 & 10.18 & 11.45 & 24.69 \\
\hline Cs3g19420 & ERF012 & 31.00 & 26.08 & 15.58 & 8.55 & 20.41 & 29.27 \\
\hline Cs3g21660 & ERF110 & 0.61 & 1.99 & 2.25 & 11.41 & 3.06 & 2.17 \\
\hline Cs6g15360 & Dehydration-responsive element-binding protein ID & 4.75 & 0.79 & 1.05 & 10.52 & 5.59 & 27.18 \\
\hline Cs6g15410 & ERF024 & 1.06 & 7.37 & 5.34 & 4.86 & 8.53 & 1.97 \\
\hline Cs8g05910 & ERF109 & 17.31 & 4.67 & 2.56 & 7.31 & 11.04 & 25.29 \\
\hline Cs9g13620 & ERF5 & 361.93 & 154.96 & 168.66 & 45.62 & 46.22 & 117.12 \\
\hline Cs4g12130 & Chitin-inducible gibberellin-responsive protein 1 & 172.13 & 61.51 & 40.99 & 54.90 & 46.03 & 92.31 \\
\hline Cs6g15680 & HRA1 & 9.24 & 2.59 & 3.18 & 1.54 & 1.76 & 4.28 \\
\hline orange1.1t00472 & WRKY46 & 224.68 & 138.04 & 83.44 & 113.21 & 101.83 & 265.74 \\
\hline
\end{tabular}

comparison groups. Therefore, we further analyzed the expression trends of the DEGs that were distributed in these five pathways and were differentially expressed in all three comparison groups. As shown in Fig. 5c and Supplementary Figure S4c, the expression of these genes exhibited significant differences between $\mathrm{Cj}$ and Pt. In the plant hormone signal transduction pathway, auxin signal genes comprised the largest group, and most of these genes $(11 / 13)$ were downregulated in Pt. The ABA, ETH, JA, CK, and SA signaling genes were also differentially expressed between $\mathrm{Cj}$ and $\mathrm{Pt}$. Moreover, several DEGs were enriched in the auxin, ABA, and JA biosynthesis pathways (Fig. $5 \mathrm{c}$ ). In the phenylpropanoid biosynthesis pathway, the expression of most of the genes $(28 / 40)$ in the $\mathrm{Pt}$ was reduced compared with that of the genes in $\mathrm{Cj}$, and most of these genes were peroxidases (Supplementary Figure S4c). These results further demonstrate that auxin, ABA, JA signal pathways, and peroxidases may play important roles in tolerance to alkaline stress.

According to the results of the $\mathrm{GO}$ enrichment analysis, "response to stimulus" was the most enriched term (Supplementary Figure S3, Table S7). A total of 118 DEGs 


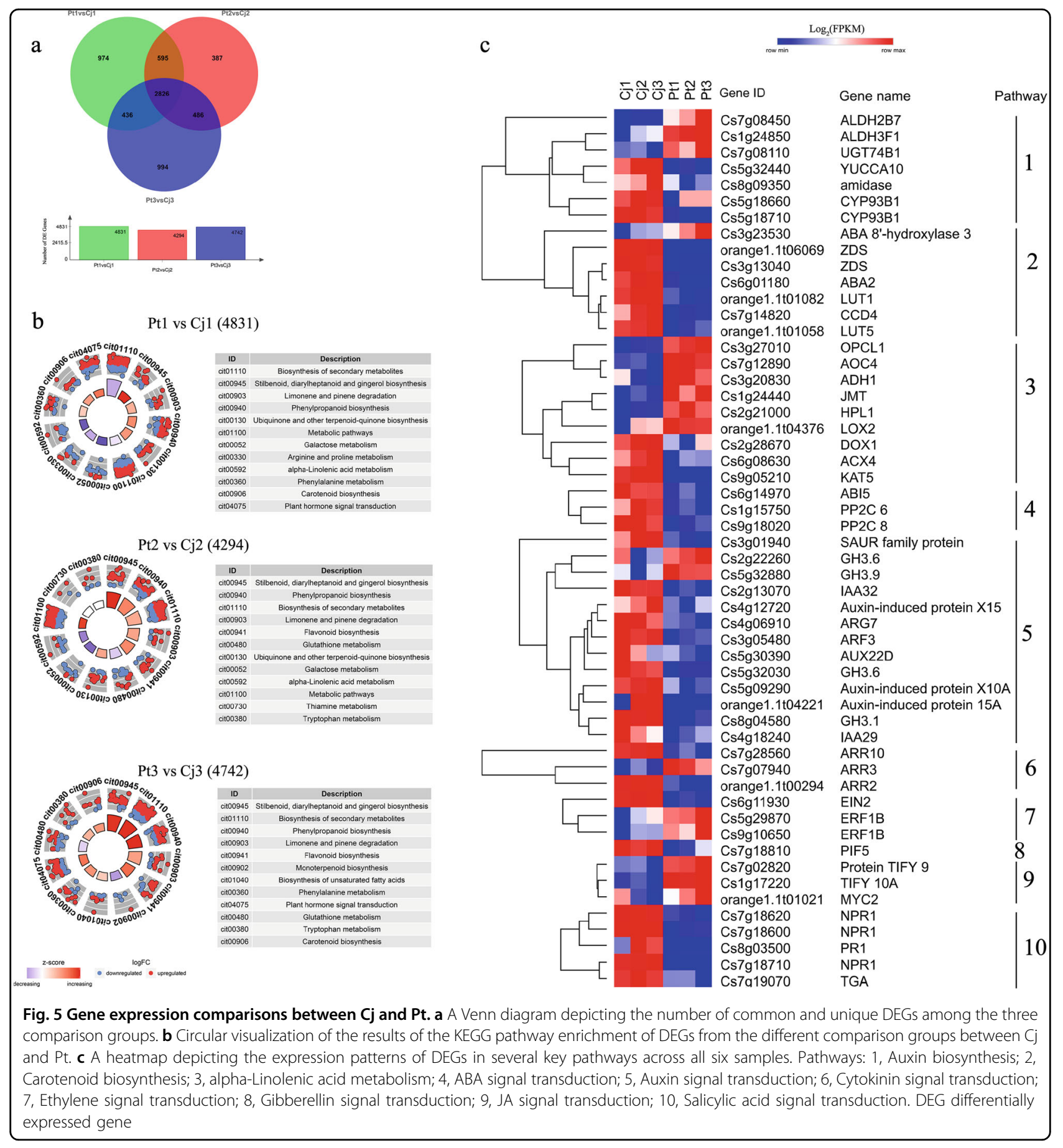

were enriched in the "response to stimulus" term in both the Pt2 vs. Cj2 and Pt3 vs. Cj3 comparison groups. As shown in Supplementary Figure S4d, the genes responding to the stimulus included disease resistance proteins; plant hormone biosynthesis and signal transduction genes; ion transport genes; and cell wall metabolism genes, such as NCED1, LOX3, FRO2/4, and peroxidases. Among these DEGs, TFs with increased expression in $\mathrm{Cj} 3$ or Pt3 included six ERF genes (ERF012, ERF110,
Dehydration-responsive element-binding protein 1D, ERF024, ERF109, and ERF5), two bHLH genes (FIT1 and ORG2), two C2H2 genes (ZFP1 and ZAT11), WRKY46, $H R A 1$ and chitin-inducible gibberellin-responsive protein 1 (Supplementary Figure S4d, Table 1). FIT1 is an essential gene that regulates iron uptake in Arabidopsis thaliana $^{37}$, and WRKY46 modulates the growth of Arabidopsis lateral roots under osmotic/salt stress conditions via regulation of $\mathrm{ABA}$ signaling and auxin homeostasis ${ }^{48}$. 
Hence, these TFs may also play important roles in citrus in response to alkaline stress.

\section{Identification and expression analysis of miRNAs in the roots of $\mathrm{Cj}$ and $\mathrm{Pt}$}

A total of 101 miRNAs (49 known miRNAs and 52 novel miRNAs) belonging to 37 families were identified from the six samples of $\mathrm{Cj}$ and Pt (Supplementary Table S8). Only the miRNAs identified in all three biological replicates were retained. All precursors of novel miRNAs exhibited regular stem-loop secondary structures. The miRNA sequences are shown in blue, and the sequences of the miRNAs* are shown in red (Supplementary Figure S5). Based on the criteria of significant difference (FDR $<0.05)$, $12,16,5,11,33,32$, and $28 \mathrm{DE}$ miRNAs (differentially expressed miRNAs) were identified in the $\mathrm{Cj} 1 \mathrm{vs.} \mathrm{Cj} 2, \mathrm{Cj} 1$ vs. Cj3, Pt2 vs. Pt3, Pt1 vs. Pt3, Pt1 vs. Cj1, Pt2 vs. Cj2, and Pt3 vs. Cj3 comparison groups, respectively (Fig. 6g, Supplementary Table S9). However, no DE miRNA was identified in the $\mathrm{Cj} 2$ vs. $\mathrm{Cj} 3$ and Pt1 vs. Pt2 comparison groups, indicating that the difference between $\mathrm{Cj} 2$ and $\mathrm{Cj} 3$ or Pt1 and Pt2 was minimal (Fig. 6g). This result was consistent with the transcriptome results (Figs. 3a, c and $6 \mathrm{~g})$. Venn diagrams and heatmaps were used to demonstrate the inclusion relations and expression patterns of DE miRNAs in these seven comparison groups (Fig. 6a-f), respectively. In total, 11 and $18 \mathrm{DE}$ miRNAs were identified among the different $\mathrm{pH}$ conditions in $\mathrm{Pt}$ and $\mathrm{Cj}$, respectively, and the number of downregulated miRNAs was much more than that of upregulated miRNAs in both Cj and Pt (Fig. 6a, b, d, e). A total of 44 DE miRNAs was identified between $\mathrm{Cj}$ and $\mathrm{Pt}$, and the expression of most of the DE miRNAs in Pt was higher than that of the DE miRNAs in $\mathrm{Cj}$ (Fig. 6c, f).

\section{Identification of PHAS genes}

From the eighteen deep sequencing sRNA libraries of $\mathrm{Cj}$ and Pt, 28 PHAS genes (the gene targeted by miRNAs to generate phasiRNAs) were identified that were capable of secondary siRNA production, with a $P$-value $\leq 0.0001$ (Supplementary Table S13). In total, 24 PHAS genes that encoded resistance proteins were targeted by the MIR482 family (csi-482-3p, csi-482b/c, and csimiR48). In addition, csi-miR393a triggered two TIR/AFB auxin receptor proteins, and csi-miR1515 triggered two dicer-like proteins (Supplementary Table S13). Moreover, the expression of csi-miR393a in Pt was higher than that in $\mathrm{Cj}$ under all three conditions (Supplementary Table S8). In this study, many genes in the auxin signal pathway were significantly altered by alkaline stress in both $\mathrm{Cj}$ and $\mathrm{Pt}$, including TIR1 (Table 1). Hence, this result suggests that csi-miR393a may be involved in the regulation of auxin signals in response to alkaline stress.

\section{Identification and functional analysis of the targets of miRNAs}

From the degradome data, a total of 260 transcripts from 150 genes were predicted to be targeted by 62 miRNAs (39 known miRNAs and 23 novel miRNAs) in $\mathrm{Cj}$, with 416 miRNA-target pairs (Supplementary Figure S6, Table S10). A total of 215 transcripts from 121 genes were predicted to be targeted by 56 miRNAs (38 known miRNAs and 18 novel miRNAs) in Pt, with 353 miRNA-target pairs (Supplementary Figure S7, Table S10). These miRNA-transcript pairs were classified into five categories (Category 0, 1, 2, 3, and 4) based on the confidence evaluation of the degradome data. In Categories 3 and 4, the miRNA-target pairs are not reliable; therefore, only those miRNA-target pairs that were distributed in Categories 0,1 , and 2 were retained. Finally, we identified 157 target genes (128 targets from $\mathrm{Cj}$ and 103 from $\mathrm{Pt}$ ) for 58 miRNAs (52 miRNAs of $\mathrm{Cj}$ and 49 miRNAs of Pt) (Supplementary Figure S8a, b).

To further elucidate the roles of miRNAs in response to alkaline stress, the targets of the DE miRNAs were used to perform GO-based term and KEGG-based pathway enrichment analyses. These targets of the DE miRNAs of each comparison group are listed in Supplementary Table S11 and are shown in Supplementary Figure S8c, d. Given that the number of targets was small in each set, no enrichment pathways were identified. According to the GO enrichment analysis results, several GO terms were highly enriched, including the response to stimulus, root cap development, lignin catabolic process, and reactive oxygen species metabolic process (Supplementary Figure S9, Table S12). The expression patterns of several important miRNAs and their targets were verified in $\mathrm{Cj}$ and Pt (Fig. 7). The targets of csi-miRN43/45 included four ARFs (ARF10/17) that are important TFs for root cap development in Arabidopsis $^{49}$ (Fig. 7a). LAC4/7/17/22 and P5CS1 are the targets of csi-miR397 (Fig. 7c). LACs are involved in lignin catabolism, and P5CS1 is a key gene for proline biosynthesis that plays important roles in scavenging reactive oxygen species $(\mathrm{ROS})^{50}$. The csimiR398 targeted several ROS scavenging genes, such as SOD1/2 and catalase (Fig. 7b). These results further suggest that the auxin pathway and ROS scavenging system may be important in the resistance to alkaline stress.

\section{Changes of phytohormone and antioxidants in $\mathrm{Cj}$ and $\mathrm{Pt}$ under alkaline stress}

According to the comprehensive analysis of transcriptomes and small RNAs of $\mathrm{Cj}$ and $\mathrm{Pt}$, the auxin, ABA, and JA signal pathways and antioxidants may play more important roles in tolerance to alkaline stress. To further verify the difference of phytohormone and antioxidants between $\mathrm{Cj}$ and $\mathrm{Pt}$ in response to alkaline stress, we measured the JA, ABA, IAA, POD, SOD, and CAT levels 




in the roots of $\mathrm{Cj}$ and $\mathrm{Pt}$ under different $\mathrm{pH}$ conditions. As shown in Fig. 8a, the IAA levels in $\mathrm{Cj}$ were significantly higher than those in $\mathrm{Pt}$ at $\mathrm{pH}$ 8.0, and no significant difference was noted between the IAA levels in $\mathrm{Cj}$ and $\mathrm{Pt}$ under the other two conditions. The ABA levels in $\mathrm{Cj}$ were significantly lower than those in Pt under all three $\mathrm{pH}$ conditions (Fig. 8b). The IAA and ABA levels decreased in both $\mathrm{Cj}$ and $\mathrm{Pt}$ as the $\mathrm{pH}$ increased. The JA levels in $\mathrm{Cj}$ were much higher than those in Pt (from approximately 12 -fold to approximately 40 -fold) under all three $\mathrm{pH}$ conditions, and the JA levels in Pt increased as the $\mathrm{pH}$ increased. By contrast, the JA levels in $\mathrm{Cj}$ were downregulated under high $\mathrm{pH}$ conditions (Fig. 8c). Regarding antioxidants, the levels of POD, SOD, and CAT decreased markedly in $\mathrm{Pt}$ as the $\mathrm{pH}$ increased; however, the levels of these antioxidants were minimally altered in $\mathrm{Cj}$ (Fig. 8d-f). These results are consistent with the results of the bioinformatics analysis.

\section{Exogenous JA and auxin analogs enhance the tolerance of} citrus to alkaline stress

To further verify the roles of JA and auxin in alkaline stress tolerance in citrus, we designed a treatment experiment using JA and auxin analogs (NAA and IBA) to treat Pt seedlings at $\mathrm{pH} 8.5$ (Fig. 9a). After 12 weeks of culture, the JA + NAA + IBA-treatment group exhibited the best growth, generating the largest number of LRs with healthy root caps and the lowest ROS level (Fig. 9). In addition, the status of the JA-treatment group was not good, which generated tiny short LRs with necrotic root caps; whereas, the ROS levels were lower than those of the control and NAA + IBA-treatment groups (Fig. 9). This result demonstrated that JA played a positive role in scavenging ROS and a negative role in LR growth and formation under alkaline stress. Moreover, the simultaneous application of auxin and JA to Pt can promote LR growth and scavenge ROS under alkaline stress. These results indicated that auxin and JA synergistically played important roles in the tolerance of citrus to alkaline stress.

\section{Discussion}

Rootstock is important for the citrus industry and can significantly affect the characteristics of scions ${ }^{39}$. Cj has increasingly been used as rootstock for many scion cultivars in recent years in China given its excellent tolerance to alkaline stress and iron deficiency. However, the 


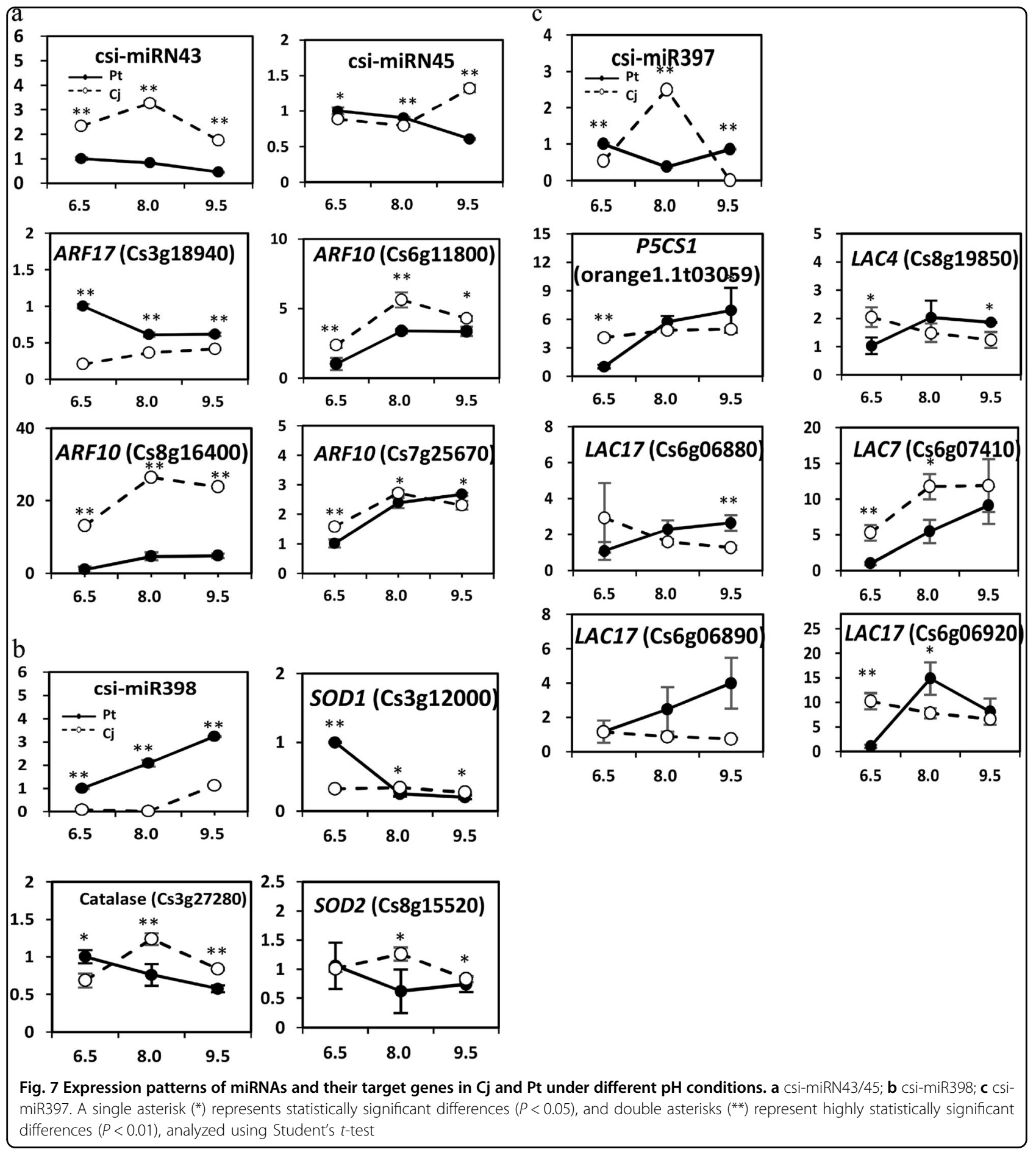

molecular mechanism has rarely been elucidated. Our work reported here provides crucial molecular insights into the tolerance of $\mathrm{Cj}$ to alkaline stress. The comprehensive transcriptomes and small RNA data sets of $\mathrm{Cj}$ and $\mathrm{Pt}$ along a $\mathrm{pH}$ gradient enabled genome-scale analyses at a high resolution. The genome-scale approach allowed us to examine the expression profiles of all of the members of gene/miRNA families in an unbiased manner and to simultaneously analyze multiple pathways. In addition, the integrated analysis of the transcriptomes and small RNAs provided more information from different regulation levels in response to alkaline stress. 

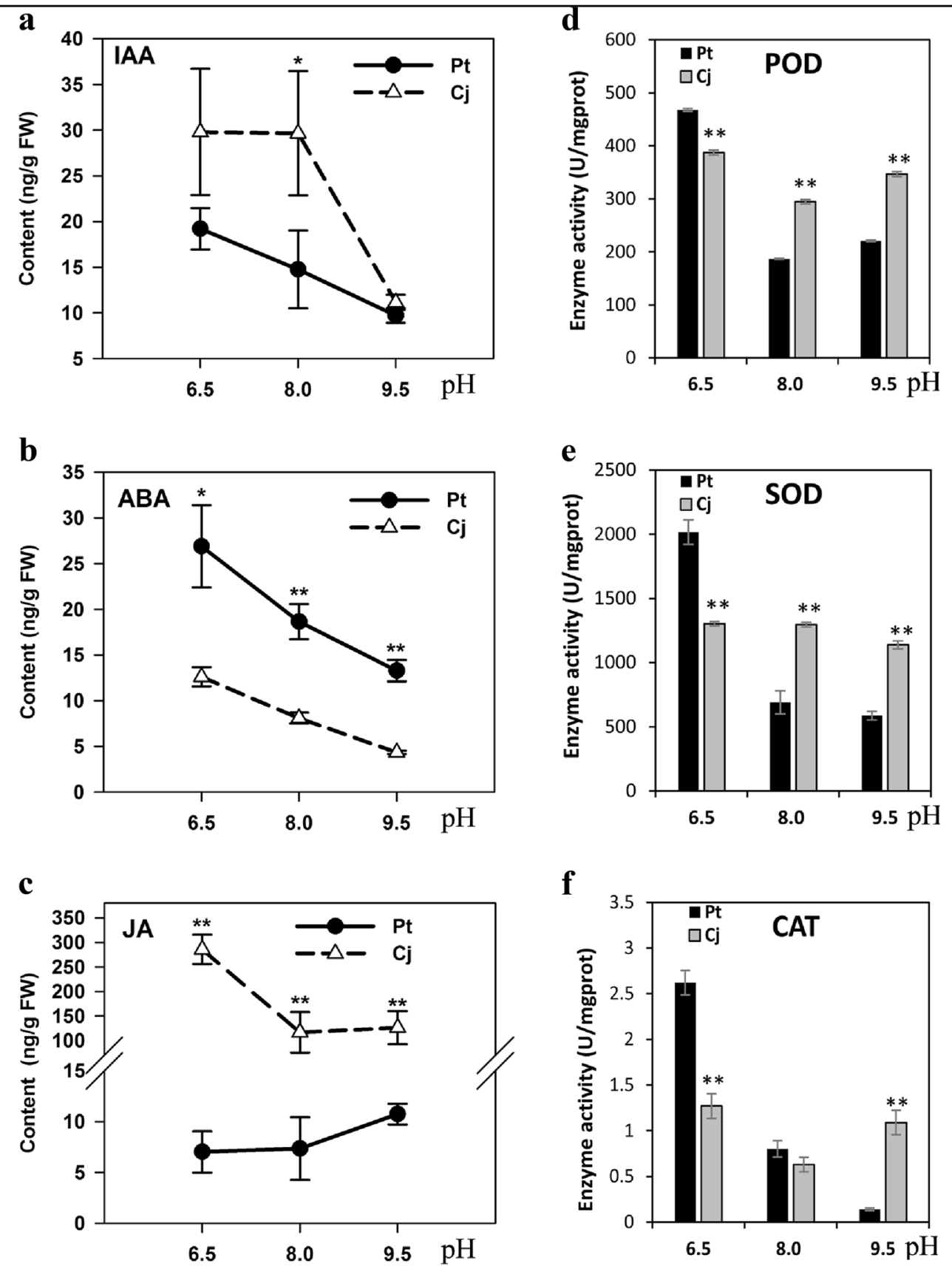

Fig. 8 Content of several types of plant hormones. a-c and antioxidants $\mathbf{d}-\mathbf{f}$ in the roots of $\mathrm{Cj}$ and $\mathrm{Pt}$ under different pH conditions. A single asterisk $\left(^{*}\right)$ represents statistically significant differences $(P<0.05)$, and double asterisks $\left(^{* *}\right)$ represent highly statistically significant differences $(P<0.01)$, analyzed using Student's $t$-test

The auxin pathway may play a central role in hormonal regulation of LR development in citrus in response to alkaline stress

In this study, alkaline stress largely inhibited the formation and growth of LR in both $\mathrm{Cj}$ and $\mathrm{Pt}$. In Arabidopsis, auxin serves as an integrator of numerous signals that are involved in LR development, such as ABA, CK, JA, and nutrients. These signals affect the LR development by interacting with auxin homeostasis (synthesis, conjugation, and degradation), transport, or response $\mathrm{e}^{9,16,29,51}$. In this study, the LR development of $\mathrm{Cj}$ and $\mathrm{Pt}$ was closely related to the content of IAA (Figs. 1a, $\mathrm{b}$ and $8 \mathrm{a}$ ). Moreover, a number of genes/miRNAs related to the auxin signal transduction pathway were differentially expressed in the roots of $\mathrm{Cj}$ and $\mathrm{Pt}$ under gradient alkaline stress, such as IAA14 (Cs1g13960), csi-miR43 and 


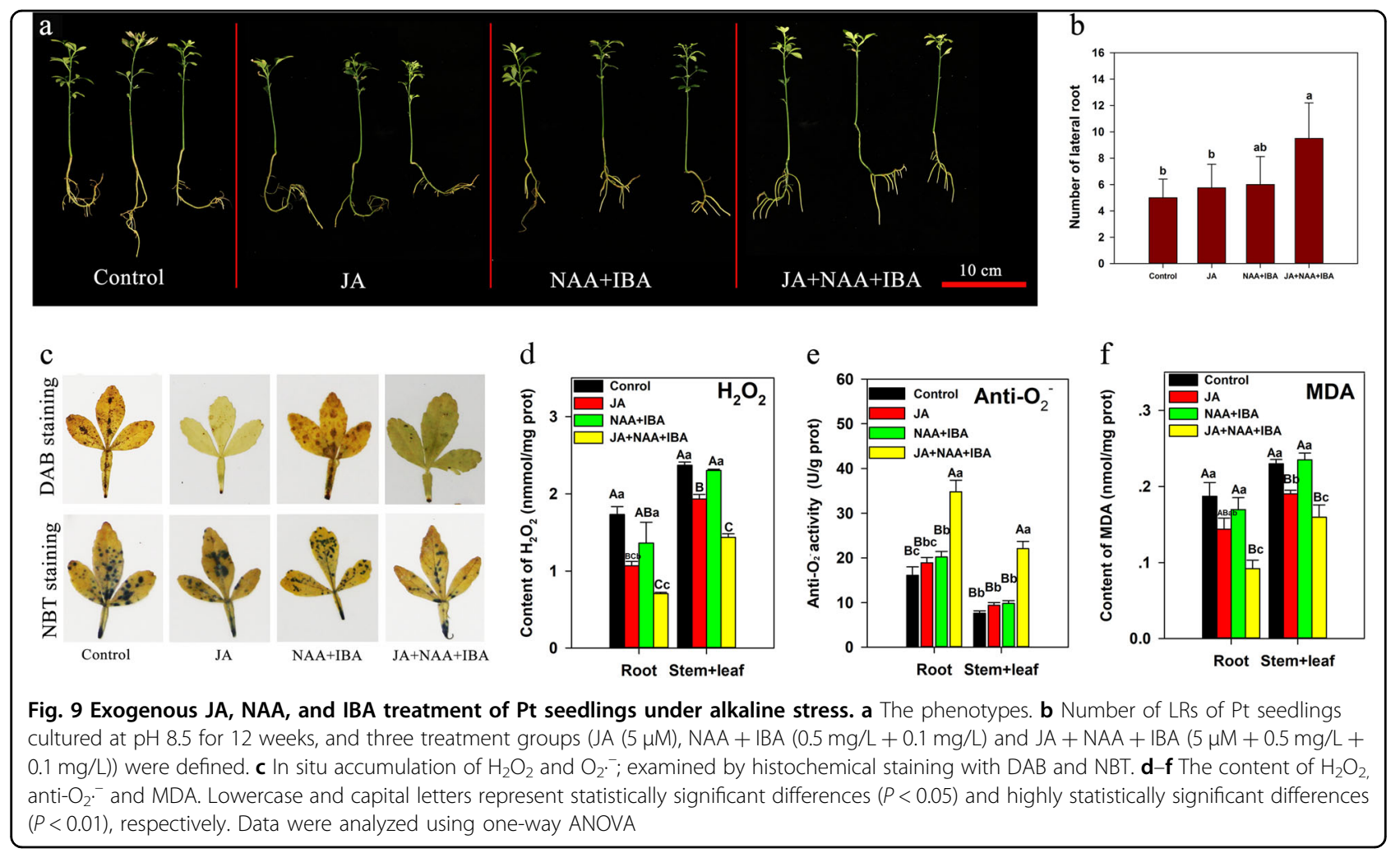

others (Figs. 4 and 6, Supplementary Figure S2). A previous study reported that IAA14 is a key regulator in auxin-regulated growth and development, particularly in lateral root formation and that the slr-1/iaa14 mutant of Arabidopsis completely lacked lateral roots ${ }^{52}$. In addition, under alkaline stress, treatment with exogenous JA, IBA, and NAA significantly increased the number of LRs (Fig. 9). These results revealed that auxin played an important role in citrus lateral root development under alkaline stress. Moreover, in this study, the expression of genes involved in IAA biosynthesis was slightly altered in $\mathrm{Cj}$ and Pt under gradient alkaline stress. However, some GH3 genes that function in conjugating amino acids to IAA $^{53-55}$ were upregulated at $\mathrm{pH} 9.5$ in $\mathrm{Cj}$ and differentially expressed between $\mathrm{Cj}$ and $\mathrm{Pt}$, such as GH3.6 (Cs2g22260) and GH3.9 (Cs5g32880) (Figs. 4 and 5c). This result suggests that the regulation of IAA homeostasis may contribute to alterations of the free IAA content in $\mathrm{Cj}$ and Pt. Overexpression GH3.6 in Arabidopsis severely reduces the number of prebranch sites and LRs $^{56}$. Moreover, several IAA-amino acid conjugates function in inhibiting root elongation, such as IAA-Ala and IAA-Leu ${ }^{57}$.

Lateral root formation and development appear to be the result of the balancing effects of auxin-promoting and ABA-repressive signaling pathways. For instance, ABA inhibits LR formation by reducing the expression of the
TIR1/AFB auxin receptor through miR393 upregulation ${ }^{58}$ and upregulating the expression of $A B I 4$, which functions to repress $P I N 1^{13}$. In this study, several key ABA biosynthesis genes were downregulated under alkaline conditions, and the ABA levels were also reduced in $\mathrm{Cj}$ and $\mathrm{Pt}$ (Figs. 2 and 8b). However, the ABA levels in Pt were higher than those in $\mathrm{Cj}$ for all three conditions (Fig. 8b). This result indicates that ABA may also play an important role in LR development under alkaline stress by interacting with the auxin pathway.

\section{Jasmonate may play a significant role in tolerance to alkaline stress}

Previous studies have demonstrated that JA is closely related to salt stress ${ }^{19,20}$; however, few studies have assessed the relationship between JA and alkaline stress. In this study, under alkaline stress, exogenous JA treatment could significantly reduce the level of $\mathrm{H}_{2} \mathrm{O}_{2}$ and MDA in Pt, and JA with auxin analogs treatment could promote LR generation and growth (Fig. 9). These results showed a positive role of JA in alkaline stress responses in citrus. However, under alkaline stress, exogenous JA treatment played a negative role in LR formation and growth in Pt (Fig. 9a, b). In addition, the expression of key genes involved in JA biosynthesis and signal transduction and the JA levels also showed opposite patterns in $\mathrm{Cj}$ and $\mathrm{Pt}$ in response to alkaline stress; these genes included 
$L O X 3$ and $M Y C 2$, which were downregulated in $\mathrm{Cj}$, but upregulated in $\mathrm{Pt}$ (Table 1). This result elucidated that JA signaling was enhanced in $\mathrm{Pt}$, but weakened in $\mathrm{Cj}$ under alkaline stress. Jasmonate-induced primary root growth inhibition has been well studied in Arabidopsis. JA reduces both the cell number and cell length of the root through $M Y C 2$ repressing the expression of PLT1 and $P L T 2^{59}$. On the other hand, JA inhibits primary root growth and regulates LR formation in a dose-dependent manner, with low micromolar concentrations of JA inhibiting the primary root growth and promoting LR formation in Arabidopsis ${ }^{30,31}$. Moreover, JA is involved in LR growth inhibition under $\mathrm{Al}$ stress and salt stress ${ }^{60,61}$. Hence, in this study, weakened JA signaling in Cj may play a positive role in the tolerance to alkaline stress. The differential response to exogenous JA may be due to the dose-dependent activity of JA.

In addition, many TFs, such as ZAT12, WRKY46, FIT1, ERF105, and others, also exhibited opposite patterns in Cj and Pt (Supplementary Figure S1d, Table 1, Supplementary Table S3). The expression patterns of these TFs were closely related with the JA levels in $\mathrm{Cj}$ and Pt. This result indicated that interactions may exist between these TFs and the JA pathway. Ding et al. ${ }^{48}$ reported that WRKY46 contributes to the feed-forward inhibition of osmotic/salt stress-dependent LR inhibition in Arabidopsis via regulation of ABA signaling and auxin homeostasis. ZAT12 is induced by $\mathrm{H}_{2} \mathrm{O}_{2}$ and functions as a negative regulator of iron acquisition by repressing FIT expression ${ }^{62}$.

\section{ROS metabolic process is important for citrus to resist alkaline stress}

When plants are exposed to stress conditions, reactive oxygen species (ROS) are generated ${ }^{63}$. A lower level of ROS following stress exposure is generally regarded as better tolerance. ROS scavenging enzymes, such as POD, SOD, and CAT, are indispensable for ROS detoxification so that plants can combat the ROS-associated cellular damage and maintain better survival under stressful conditions $^{64}$. In this study, the expression of genes/ miRNAs of ROS metabolic process were affected by alkaline stress in $\mathrm{Cj}$ and $\mathrm{Pt}$, such as peroxidase genes and csi-miR398 (Figs. 4 and 7b, Supplementary Figure S2). Moreover, most ROS metabolic process genes/miRNAs were more downregulated/upregulated in Pt than those in $\mathrm{Cj}$ in response to alkaline stress (Figs. 4 and $7 \mathrm{~b}$, Supplementary Figure S2). In addition, the SOD, POD, and CAT levels were also much higher in $\mathrm{Cj}$ than those in Pt under alkaline stress, and these antioxidant levels changed slightly in $\mathrm{Cj}$ in response to alkaline stress (Fig. 8d-f). Therefore, these results showed the ROS scavenging system of $\mathrm{Cj}$ was stronger than that of Pt under alkaline stress. Several studies have shown that exogenous JA treatment of soybean significantly increases the activities of SOD, POD, APX, and CAT under salt or drought stress $^{65,66}$. In this study, under alkaline stress, exogenous JA treatment could significantly reduce the levels of MDA and $\mathrm{H}_{2} \mathrm{O}_{2}$ in Pt seedlings (Fig. 9d, f). Hence, the interaction between JA and ROS metabolic process may play roles in the tolerance of citrus to alkaline stress.

\section{Differential response of $\mathrm{Cj}$ and $\mathrm{Pt}$ to iron deficiency under alkaline stress}

$\mathrm{Cj}$ is more tolerant to iron deficiency compared with $\mathrm{Pt}$ under alkaline stress. In this study, a number of genes related to iron acquisition were differentially expressed among the different $\mathrm{pH}$ conditions in $\mathrm{Cj}$ and $\mathrm{Pt}$, including FIT1 and FRO2/4/6 (Table 1). Moreover, these genes were upregulated in $\mathrm{Cj}$ but downregulated in $\mathrm{Pt}$ as the $\mathrm{pH}$ increased (Table 1, Supplementary Figure S4a, b). This result indicates improved iron acquisition in $\mathrm{Cj}$ compared with Pt under alkaline stress. Previous studies demonstrated that FIT plays a central role in iron acquisition ${ }^{38}$. FRO2 and IRT1 are targets of FIT that regulate FRO2 at the level of mRNA accumulation and IRT1 at the level of protein accumulation $^{37}$. FIT expression is upregulated by iron deficiency ${ }^{37}$ and is downregulated by jasmonate ${ }^{67,68}$. In this study, the JA levels were decreased in $\mathrm{Cj}$ but increased in $\mathrm{Pt}$ as the $\mathrm{pH}$ increased (Fig. 8c). This result suggests that $J A$ may repress $F I T 1$ expression in citrus under alkaline stress. The interaction between FIT1 and JA should be studied in the future.

Taken together, our results demonstrate that the inhibitory effect of alkaline stress on root growth involves the auxin, ABA and JA signaling pathways in citrus. Regulation of auxin homeostasis under alkaline stress is important for adapting to alkaline stress in citrus. Moreover, the JA pathway, which exhibits the opposite response to alkaline stress in $\mathrm{Cj}$ and $\mathrm{Pt}$, may contribute to the differences in the alkaline stress and iron deficiency tolerance between $\mathrm{Cj}$ and Pt. Further studies are needed to elucidate the regulation network of JA in resistance to alkaline stress and iron deficiency in citrus.

\section{Materials and methods}

Plant materials, growth conditions, and sample collections

According to the method described by Zhou et al. ${ }^{69}$, the seeds of $\mathrm{Cj}$ and $\mathrm{Pt}$ were surface sterilized, and their germination was accelerated. The seedlings were grown in a growth chamber until they had four leaves. Then, seedlings were selected based on a uniform size and transferred into a hydroponics system with a $4 \mathrm{~L}$ solution in a greenhouse. Three different $\mathrm{pH}$ gradients $(6.5,8.0$, and 9.5) were established. Given that most natural soils have weak acidity, we used $\mathrm{pH} 6.5$ as the normal condition. After 8 weeks of culture, three biological replicates (three seedling plants per replicate) were collected randomly for each treatment. $\mathrm{Cj} 1, \mathrm{Cj} 2$, and $\mathrm{Cj} 3$ represented $\mathrm{Cj}$ seedlings 
cultured at $\mathrm{pH} 6.5,8.0$, and 9.5, respectively. A similar naming scheme was employed for the Pt seedlings. The roots, stems, and leaves of the seedlings were sampled separately. A portion of root samples was scanned with an Epson digital scanner (Expression 10000XL 1.0, Epson, Inc., Japan), and WinRhizo Pro (S) v. 2009c (Regent Instruments, Inc., Canada) software was used to analyze the root morphology. Another portion of samples were frozen by liquid nitrogen and immediately stored under $-80^{\circ} \mathrm{C}$ for other experiments.

\section{Measurement of mineral element concentrations}

The plant mineral element concentrations were measured as described in previous studies ${ }^{69,70}$, with some modification. Briefly, fresh samples were placed into a forced air oven at $105^{\circ} \mathrm{C}$ for $30 \mathrm{~min}$, and then at $75^{\circ} \mathrm{C}$ until a constant weight was reached to determine the sample dry weight. All the dried samples were ground into fine powder. Then, $0.50 \mathrm{~g}$ of each sample was dry-ashed in a muffle furnace at $200{ }^{\circ} \mathrm{C}$ for $1 \mathrm{~h}, 300^{\circ} \mathrm{C}$ for $1 \mathrm{~h}$, and $500{ }^{\circ} \mathrm{C}$ for $8 \mathrm{~h}$, followed by dissolution in $10 \mathrm{~mL} 0.1 \mathrm{~N} \mathrm{HCl}$. The mineral elements (except nitrogen) were determined using inductively coupled plasma atomic spectroscopy (ICP-AES; Thermo, Inc., IRIS Advan, USA). The total nitrogen content of the plant samples, the $\mathrm{pH}$ of the soil samples and the soil mineral element concentrations were measured using the methods described by $\mathrm{Bao}^{71}$. Each sample was assayed using three replicates.

\section{RNA-seq, data processing, and gene annotation}

Total RNA isolation was performed as described previously $^{72}$. Six samples of the root tissues of $\mathrm{Cj}$ and Pt were collected, and three biological replicates were collected for each sample. A total of 18 transcriptomes, 18 small RNAs and two degradomes profiles were obtained by RNA-seq using Illumina HiSeq X-ten at Beijing Biomarker Technologies Limited Company (Beijing) in 2017. The sequencing raw data have been submitted to NCBI Gene Expression Omnibus (GEO), and the accession number is GSE115050. The method for data processing and gene annotation was listed in Supplementary Methods 1 .

\section{Expression data analysis}

The dendrogram in Fig. 2a was made by the function cor () in $\mathrm{R}$ with default settings. The $y$-axis is computed as 1 minus cor (correlation) to reflect the degree of variance. $\log _{2}$ (FPKM) was used in the computation. The expression modules in Fig. 2c, d were generated by the Short Time-series Expression Miner (v1.3.7) (STEM) ${ }^{45}$ based on FPKM, with the STEM Clustering Method, with default settings used for the Filtering, Model Profiles and Clustering Profiles. Figure $3 \mathrm{~b}, \mathrm{~d}$ was generated by the function GOCircle () of R package GOplot (1.0.2) ${ }^{46}$. Hierarchical clustering of the gene sets was performed using Morpheus (https://software.broadinstitute.org/morpheus/) with one minus the Pearson correlation as the distance metric and average as the linkage method. $\log _{2}$ (FPKM) of the differentially expressed genes from the comparison groups was used for clustering. The $Z$-score in here was calculated for each miRNA per sample using the formula $\left(X-X_{\text {av }}\right) / \mathrm{SD}$, where $X$ is the TPM value in a particular sample, and $X_{\mathrm{av}}$ and $\mathrm{SD}$ are the mean and standard deviation of the TPM values across all samples used for clustering, respectively.

\section{Verification of miRNA and target gene expression by qRT-PCR}

Stem-loop qRT-PCR was performed to validate the expressions of miRNAs with three biological replicates based on our previous method ${ }^{73}$. U6 was used as the endogenous reference gene ${ }^{74}$. Next, qRT-PCR was performed with three biological replicates according to our previous study ${ }^{75}$, and CsActin was used as the endogenous reference gene ${ }^{76}$. The primers are listed in Supplementary Table S14. Data are presented as the means \pm standard error $(\mathrm{SE})(n=3)$.

\section{Quantification of $A B A, J A$, and IAA}

The samples for ABA, JA, and IAA quantification were prepared according to the method described by Pan et al. ${ }^{77}$, with slight modifications, which were described in detail in our previous study ${ }^{75}$. For this purpose, $d_{6}-A B A$ (Icon Isotopes, cat. no. ID1001) was used as an internal standard for ABA, $d_{5}$-IAA (Aldrich, cat. no. 492817) was used as an internal standard for IAA, and $\mathrm{H}_{2}$ JA (dihydrojasmonic acid, OlChemim, cat. no. 0145324) was used as an internal standard for JA. The reaction monitoring conditions $(\mathrm{Q} 1 / \mathrm{Q} 3)$ for $\mathrm{ABA}$ and $\mathrm{d}_{6}-\mathrm{ABA}$, IAA and $\mathrm{d}_{5^{-}}$ IAA, and JA and $\mathrm{H}_{2}$ JA were 262.8/152.6 and 269.1/158.8, 174.0/129.6 and 179.0/134.8, and 209.0/59.0 and 211.0/ 58.8, respectively. Each sample was characterized using four replicates.

\section{Physiological analyses and histochemical staining of ROS}

Malondialdehyde (MDA) levels were determined according to the method described by Liu et al. ${ }^{78}$. Peroxidase (POD), superoxide dismutase (SOD), catalase (CAT) and $\mathrm{H}_{2} \mathrm{O}_{2}$ were measured using relevant detection kits (A064-1 for $\mathrm{H}_{2} \mathrm{O}_{2}$, A084-3 for POD, A001-1 for SOD, and A007-1 for CAT, Nanjing Jiancheng Bioengineering Institute, Jiangsu, China) following the manufacturer's instructions. Anti- $\mathrm{O}_{2}{ }^{-}$measurement and total protein concentration measurement were performed according to Geng and $\mathrm{Liu}^{64}$. Histochemical staining with 3,3'-diaminobenzidine $(\mathrm{DAB})$ and nitroblue tetrazolium $(\mathrm{NBT})$ was used to examine the in situ accumulation of $\mathrm{H}_{2} \mathrm{O}_{2}$ and $\mathrm{O}_{2}{ }^{-}{ }^{-}$, respectively ${ }^{79}$. 


\section{JA, naphthylacetic acid (NAA), and indolebutyric acid (IBA) treatment of the seedlings of $\mathrm{Cj}$ and $\mathrm{Pt}$}

A stock solution of $1 \mathrm{mM} \mathrm{JA}$ was made in ethanol and diluted with water before application. For the Pt seedlings cultured at $\mathrm{pH} 8.5$, we set up the JA $(5 \mu \mathrm{M})$, NAA + IBA $(0.5 \mathrm{mg} / \mathrm{L}+0.1 \mathrm{mg} / \mathrm{L})$ and JA + NAA + IBA $(5 \mu \mathrm{M}+$ $0.5 \mathrm{mg} / \mathrm{L}+0.1 \mathrm{mg} / \mathrm{L}$ ) treatment groups, and the seedlings were cultured for 12 weeks. The solution was replaced every week.

\section{Acknowledgements}

This research was supported by the National Modern Citrus Industry System (CARS-26), NSFC (Natural Science Foundation of China) (31601729), NSFC (31521092) and the China Postdoctoral Science Foundation (2015M582242). We thank Yuan Zhou (a graduate of the College of Resources and Environment) for her help in the determination of the content of nutrition elements in the soil.

\section{Conflict of interest}

The authors declare that they have no conflict of interest.

\section{Publisher's note}

Springer Nature remains neutral with regard to jurisdictional claims in published maps and institutional affiliations.

Supplementary Information accompanies this paper at (https://doi.org/ 10.1038/s41438-018-0116-0)

Received: 3 June 2018 Revised: 8 October 2018 Accepted: 14 October 2018 Published online: 01 March 2019

\section{References}

1. Ge, Y. et al. Global transcriptome profiling of wild soybean (Glycine soja) roots under $\mathrm{NaHCO}_{3}$ treatment. BMC Plant Biol. 10, https:/doi.org/10.1186/14712229-10-153 (2010).

2. $\mathrm{Xu}, \mathrm{W}$. F. et al. PIN2 is required for the adaptation of Arabidopsis roots to alkaline stress by modulating proton secretion. J. Exp. Bot. 63, 6105-6114 (2012).

3. Xu, W. F. et al. The tomato 14-3-3 protein TFT4 modulates H+Efflux, basipetal auxin transport, and the PKS5-J3 pathway in the root growth response to alkaline stress. Plant Physiol. 163, 1817-1828 (2013).

4. Jin, H. et al. Comparative EST profiles of leaf and root of Leymus chinensis, a xerophilous grass adapted to high pH sodic soil. Plant Sci. 170, 1081-1086 (2006).

5. Wang, Y. C. et al. Analysis of gene expression profile of limonium bicolor under $\mathrm{NaHCO}_{3}$ stress using cDNA microarray. Plant Mol. Biol. Rep. 26, 241-254 (2008).

6. Yang, C. W., Wang, P., Li, C. Y., Shi, D. C. \& Wang, D. L. Comparison of effects of salt and alkali stresses on the growth and photosynthesis of wheat. Photosynthetica 46, 107-114 (2008).

7. Zhu, J. K. Salt and drought stress signal transduction in plants. Annu. Rev. Plant Biol. 53, 247-273 (2002).

8. Den Herder, G., Van Isterdael, G., Beeckman, T. \& De Smet, I. The roots of a new green revolution. Trends Plant. Sci. 15, 600-607 (2010).

9. Lavenus, J. et al. Lateral root development in Arabidopsis: fifty shades of auxin. Trends Plant Sci. 18, 450-458 (2013).

10. Laplaze, L. et al. Cytokinins act directly on lateral root founder cells to inhibit root initiation. Plant Cell 19, 3889-3900 (2007).

11. Moriwaki, T. et al. Hormonal regulation of lateral root development in arabidopsis modulated by MIZ1 and requirement of GNOM activity for MIZ1 function. Plant Physiol. 157, 1209-1220 (2011).

12. Chen, H., Li, Z. F. \& Xiong, L. M. A plant microRNA regulates the adaptation of roots to drought stress. FEBS Lett. 586, 1742-1747 (2012).
13. Shkolnik-Inbar, D. \& Bar-Zvi, D. ABI4 mediates abscisic acid and cytokinin inhibition of lateral root formation by reducing polar auxin transport in Arabidopsis. Plant Cell 22, 3560-3573 (2010).

14. Gifford, M. L., Dean, A., Gutierrez, R. A., Coruzzi, G. M. \& Birnbaum, K. D. Cellspecific nitrogen responses mediate developmental plasticity. Proc. Natl Acad. Sci. USA 105, 803-808 (2008)

15. Shin, R. et al. The Arabidopsis transcription factor MYB77 modulates auxin signal transduction. Plant Cell 19, 2440-2453 (2007).

16. Perez-Torres, C. A. et al. Phosphate availability alters lateral root development in Arabidopsis by modulating auxin sensitivity via a mechanism involving the TIR1 auxin receptor. Plant Cell 20, 3258-3272 (2008).

17. Creelman, R. A. \& Mullet, J. E. Jasmonic acid distribution and action in plants: regulation during development and response to biotic and abiotic stress. Proc. Natl Acad. Sci. USA 92, 4114-4119 (1995).

18. Wasternack, C. Jasmonates: an update on biosynthesis, signal transduction and action in plant stress response, growth and development. Ann. Bot. 100, 681-697 (2007).

19. Pedranzani, $\mathrm{H}$. et al. Salt tolerant tomato plants show increased levels of jasmonic acid. Plant Growth Regul. 41, 149-158 (2003).

20. Kang, D. J. et al. Jasmonic acid differentially affects growth, ion uptake and abscisic acid concentration in salt-tolerant and salt-sensitive rice cultivars. J. Agron. Crop Sci. 191, 273-282 (2005).

21. Yoon, J. Y., Hamayun, M., Lee, S.-K. \& Lee, I.-J. Methyl jasmonate alleviated salinity stress in soybean. J. Crop Sci. Biotechnol. 12, 63-68 (2009).

22. Javid, M. G., Sorooshzadeh, A., Moradi, F., Sanavy, S. A. M. M. \& Allahdadi, I. The role of phytohormones in alleviating salt stress in crop plants. Aust. J. Crop Sci. 5, 726-734 (2011)

23. Qiu, Z. B., Guo, J. L., Zhu, A. J., Zhang, L. \& Zhang, M. M. Exogenous jasmonic acid can enhance tolerance of wheat seedlings to salt stress. Ecotoxicol. Environ. Saf. 104, 202-208 (2014).

24. Ismail, A., Riemann, M. \& Nick, P. The jasmonate pathway mediates salt tolerance in grapevines. J. Exp. Bot. 63, 2127-2139 (2012).

25. Wu, H., Ye, H. Y., Yao, R. F., Zhang, T. \& Xiong, L. Z. OsJAZ9 acts as a transcriptional regulator in jasmonate signaling and modulates salt stress tolerance in rice. Plant Sci. 232, 1-12 (2015).

26. Zhu, D. et al. The positive regulatory roles of the TIFY10 proteins in plant responses to alkaline stress. PLOS ONE 9, https://doi.org/10.1371/journal. pone.0111984 (2014).

27. Zhu, D. et al. Over-expression of a novel JAZ family gene from Glycine soja, increases salt and alkali stress tolerance. Biochem. Biophys. Res. Commun. 426, 273-279 (2012).

28. Yan, Y. et al. A downstream mediator in the growth repression limb of the jasmonate pathway. Plant Cell 19, 2470-2483 (2007)

29. Sun, J. Q. et al. Arabidopsis ASA1 is important for jasmonate-mediated regulation of auxin biosynthesis and transport during lateral root formation. Plant Cell 21, 1495-1511 (2009).

30. Sun, J. Q. et al. Jasmonate modulates endocytosis and plasma membrane accumulation of the Arabidopsis PIN2 protein. New Phytol. 191, 360-375 (2011).

31. Raya-Gonzalez, J., Pelagio-Flores, R. \& Lopez-Bucio, J. The jasmonate receptor COI1 plays a role in jasmonate-induced lateral root formation and lateral root positioning in Arabidopsis thaliana. J. Plant Physiol. 169, 1348-1358 (2012)

32. Toda, Y. et al. RICE SALT SENSITIVE3 forms a ternary complex with JAZ and Class-C bHLH factors and regulates jasmonate-induced gene expression and root cell elongation. Plant Cell 25, 1709-1725 (2013).

33. Robinson, N. J., Procter, C. M., Connolly, E. L. \& Guerinot, M. L. A ferric-chelate reductase for iron uptake from soils. Nature 397, 694-697 (1999).

34. Connolly, E. L., Campbell, N. H., Grotz, N., Prichard, C. L. \& Guerinot, M. L. Overexpression of the FRO2 ferric chelate reductase confers tolerance to growth on low iron and uncovers posttranscriptional control. Plant Physiol. 133, 1102-1110 (2003).

35. Eide, D., Broderius, M., Fett, J. \& Guerinot, M. L. A novel iron-regulated metal transporter from plants identified by functional expression in yeast. Proc. Nat Acad. Sci. USA 93, 5624-5628 (1996).

36. Vert G. et al. IRT1, an Arabidopsis transporter essential for iron uptake from the soil and for plant growth. Plant Cell 14, 1223-1233 (2002).

37. Colangelo, E. P. \& Guerinot, M. L. The essential basic helix-loop-helix protein FIT1 is required for the iron deficiency response. Plant Cell 16, 3400-3412 (2004). 
38. Yuan, Y. X., Zhang, J., Wang, D. W. \& Ling, H. Q. AtbHLH29 of Arabidopsis thaliana is a functional ortholog of tomato FER involved in controlling iron acquisition in strategy I plants. Cell Res. 15, 613-621 (2005).

39. Castle, W. S. Rootstock as a fruit quality factor in citrus and deciduous tree crops. N.Z. J. Crop Hortic. Sci. 23, 383-394 (1995).

40. Xu, Q. et al. The draft genome of sweet orange (Citrus sinensis). Nat. Genet. 45, 59-U92 (2013).

41. Zhang, Z., Jiang, L., Wang, J., Gu, P. \& Chen, M. MTide: an integrated tool for the identification of miRNA-target interaction in plants. Bioinformatics 31, 290-291 (2015).

42. Meyers, B. C. et al. Criteria for annotation of plant MicroRNAs. Plant Cell 20, 3186-3190 (2008).

43. German, M. A. et al. Global identification of microRNA-target RNA pairs by parallel analysis of RNA ends. Nat. Biotechnol. 26, 941-946 (2008).

44. Addo-Quaye, C., Eshoo, T. W., Bartel, D. P. \& Axtell, M. J. Endogenous siRNA and miRNA targets identified by sequencing of the Arabidopsis degradome. Curr. Biol. 18, 758-762 (2008).

45. Ernst, J. \& Bar-Joseph, Z. STEM: a tool for the analysis of short time series gene expression data. BMC Bioinform. 7, 191 (2006).

46. Walter, W., Sanchez-Cabo, F. \& Ricote, M. GOplot: an R package for visually combining expression data with functional analysis. Bioinformatics $\mathbf{3 1}$, 2912-2914 (2015).

47. Wittstock, U. \& Halkier, B. A. Cytochrome P450 CYP79A2 from Arabidopsis thaliana L. Catalyzes the conversion of L-phenylalanine to phenylacetaldoxime in the biosynthesis of benzylglucosinolate. J. Biol. Chem. 275, 14659-14666 (2000).

48. Ding, Z. J. et al. Transcription factor WRKY46 modulates the development of Arabidopsis lateral roots in osmotic/salt stress conditions via regulation of $A B A$ signaling and auxin homeostasis. Plant J. 84, 56-69 (2015).

49. Wang, J. W. et al. Control of root cap formation by microRNA-targeted auxin response factors in Arabidopsis. Plant Cell 17, $2204-2216$ (2005).

50. Verbruggen, N. \& Hermans, C. Proline accumulation in plants: a review. Amino Acids 35, 753-759 (2008)

51. Bishopp, A., Benkova, E. \& Helariutta, Y. Sending mixed messages: auxincytokinin crosstalk in roots. Curr. Opin. Plant Biol. 14, 10-16 (2011).

52. Hidehiro, F., Satoshi, T., Haruka, M. \& Masao, T. Lateral root formation is blocked by a gain-of-function mutation in the SOLITARY-ROOT/IAA14 gene of Arabidopsis. Plant J. 29, 153-168 (2002).

53. Khan, S. \& Stone, J. M. Arabidopsis thaliana GH3.9 in Auxin and Jasmonate Cross Talk. Plant Signal. Behav. 2, 483-485 (2007).

54. Staswick, P. E. et al. Characterization of an Arabidopsis enzyme family that conjugates amino acids to indole-3-acetic acid. Plant Cell 17, 616-627 (2005).

55. Gutierrez, L. et al. Auxin controls Arabidopsis adventitious root initiation by regulating jasmonic acid homeostasis. Plant Cell 24, 2515-2527 (2012).

56. Xuan, W. et al. Root cap-derived auxin pre-patterns the longitudinal axis of the Arabidopsis root. Curr. Biol. 25, 1381-1388 (2015).

57. LeClere, S., Tellez, R., Rampey, R. A., Matsuda, S. P. T. \& Bartel, B. Characterization of a family of IAA-amino acid conjugate hydrolases from Arabidopsis. J. Biol. Chem. 277, 20446-20452 (2002).

58. Vidal, E. A. et al. Nitrate-responsive miR393/AFB3 regulatory module controls root system architecture in Arabidopsis thaliana. Proc. Natl Acad. Sci. USA 107, 4477-4482 (2010).

59. Chen, Q. et al. The basic helix-loop-helix transcription factor MYC2 directly represses PLETHORA expression during jasmonate-mediated modulation of the root stem cell niche in Arabidopsis. Plant Cell 23, 3335-3352 (2011).

60. Yang, Z. B., He, C. M., Ma, Y. Q., Herde, M. \& Ding, Z. J. Jasmonic acid enhances al-induced root growth inhibition. Plant Physiol. 173, 1420-1433 (2017).
61. Valenzuela, C. E. et al. Salt stress response triggers activation of the jasmonate signaling pathway leading to inhibition of cell elongation in Arabidopsis primary root. J. Exp. Bot. 67, 4209-4220 (2016).

62. Le, C. T. T. et al. ZINC FINGER OF ARABIDOPSIS THALIANA12 (ZAT12) interacts with FER-LIKE IRON DEFICIENCY- INDUCED TRANSCRIPTION FACTOR (FIT) linking iron deficiency and oxidative stress responses. Plant Physiol. 170 540-557 (2016).

63. Gill, S. S. \& Tuteja, N. Reactive oxygen species and antioxidant machinery in abiotic stress tolerance in crop plants. Plant Physiol. Biochem. 48, 909-930 (2010).

64. Geng, J. J. \& Liu, J. H. The transcription factor CsbHLH18 of sweet orange functions in modulation of cold tolerance and homeostasis of reactive oxygen species by regulating the antioxidant gene. J. Exp. Bot. 69 2677-2692 (2018).

65. Anjum, S. A., Wang, L., Farooq, M., Khan, I. \& Xue, L. Methyl jasmonate-induced alteration in lipid peroxidation, antioxidative defence system and yield in soybean under drought. J. Agron. Crop Sci. 197, 296-301 (2011).

66. Noriega, G., Cruz, D. S., Batlle, A., Tomaro, M. \& Balestrasse, K. Heme oxygenase is involved in the protection exerted by jasmonic acid against cadmium stress in soybean roots. J. Plant Growth Regul. 31, 79-89 (2012).

67. Maurer, F., Müller, S. \& Bauer, P. Suppression of Fe deficiency gene expression by jasmonate. Plant Physiol. Biochem. 49, 530-536 (2011).

68. Brumbarova, T., Bauer, P. \& Ivanov, R. Molecular mechanisms governing Arabidopsis iron uptake. Trends Plant Sci. 20, 124-133 (2015).

69. Zhou, G. F. et al. Transcription profiles of boron-deficiency-responsive genes in citrus rootstock root by suppression subtractive hybridization and CDNA microarray. Front. Plant Sci. 5, https://doi.org/10.3389/Fpls.2014.00795 (2015).

70. Storey, R. \& Treeby, M. T. Seasonal changes in nutrient concentrations of navel orange fruit. Sci. Hortic. 84, 67-82 (2000).

71. Bao, S. D. Soil agrochemical analysis. third edn, (China Agriculture Press (in (hinese), 2008).

72. Liu, Y., Liu, Q. \& Tao, N. Efficient isolation of RNA from fruit peel and pulp of ripening navel orange (Citrus sinensis Osbeck). J. Huazhong Agric. Univ. 25, 300-304 (2006).

73. Wu, J. X., Zheng, S. S., Feng, G. Z. \& Yi, H. L. Comparative analysis of miRNAs and their target transcripts between a spontaneous late-ripening sweet orange mutant and its wild-type using small rna and degradome sequencing. Front. Plant Sci. 7, https://doi.org/10.3389/fpls.2016.01416 (2016).

74. Kou, S. J. et al. Selection and validation of suitable reference genes for miRNA expression normalization by quantitative RT-PCR in citrus somatic embryogenic and adult tissues. Plant Cell Rep. 31, 2151-2163 (2012).

75. $\mathrm{Wu}$, J. et al. An integrative analysis of the transcriptome and proteome of the pulp of a spontaneous late-ripening sweet orange mutant and its wild type improves our understanding of fruit ripening in citrus. J. Exp. Bot. 65, 1651-1671 (2014)

76. $\mathrm{Wu}$, J. et al. Selection of reliable reference genes for gene expression studies using quantitative real-time PCR in navel orange fruit development and pummelo floral organs. Sci. Hortic. 176, 180-188 (2014).

77. Pan, X., Welti, R. \& Wang, X. Quantitative analysis of major plant hormones in crude plant extracts by high-performance liquid chromatography-mass spectrometry. Nat. Protocol 5, 986-992 (2010)

78. Liu, J. H. et al. Polyamine biosynthesis of apple callus under salt stress: importance of the arginine decarboxylase pathway in stress response. J. Exp. Bot. 57, 2589-2599 (2006)

79. Wang, J. et al. An arginine decarboxylase gene PtADC from Poncirus trifoliata confers abiotic stress tolerance and promotes primary root growth in Arabidopsis. J. Exp. Bot. 62, 2899-2914 (2011). 